\title{
Trace Inclusion for One-Counter Nets Revisited
}

\author{
Piotr Hofman ${ }^{1}$ and Patrick Totzke ${ }^{2}$ \\ 1 University of Bayreuth, Germany \\ ${ }^{2}$ LaBRI, Université de Bordeaux
}

\begin{abstract}
One-counter nets $(\mathrm{OCN})$ consist of a nondeterministic finite control and a single integer counter that cannot be fully tested for zero. They form a natural subclass of both One-Counter Automata, which allow zero-tests and Petri Nets/VASS, which allow multiple such weak counters. The trace inclusion problem has recently been shown to be undecidable for OCN. In this paper, we contrast the complexity of two natural restrictions which imply decidability.

First, we show that trace inclusion between an OCN and a deterministic OCN is NL-complete, even with arbitrary binary-encoded initial counter-values as part of the input. Secondly, we show Ackermannian completeness of for the trace universality problem of nondeterministic OCN. This problem is equivalent to checking trace inclusion between a finite and a OCN-process.
\end{abstract}

\section{Introduction}

A fundamental question in formal verification is if the behaviour of one process can be reproduced by - or equals that of - another given process. These inclusion and equivalence problems, respectively have been studied for various notions of behavioural preorders and equivalences and for many computational models. Trace inclusion/equivalence asks if the set of traces, all emittable sequences of actions, of one process is contained in/equal to that of another. Other than for instance Simulation preorder, trace inclusion lacks a strong locality of failures, which makes this problem intractable or even undecidable already for very limited models of computation.

We consider one-counter nets, which consist of a finite control and a single integer counter that cannot be fully tested for zero, in the sense that an empty counter can only restrict possible moves. They are subsumed by One-counter automata (OCA) and thus Pushdown Systems, which allow explicit zero-tests by reading a bottom marker on the stack. At the same time, OCN are a subclass of Petri Nets or Vector Addition Systems with states (VASS): they are exactly the one-dimensional VASS and thus equivalent to Petri Nets with at most one unbounded place.

Related work. Valiant and Paterson [15] show the decidability of the trace equivalence problem for deterministic one-counter automata (DOCA). This problem has recently been shown to be NL-complete by Böhm, Göller, and Jančar 
[2], assuming fixed initial counter-values. The equivalence of deterministic pushdown automata is known to be decidable [11] and primitive recursive [12], but the exact complexity is still open.

Valiant [14] proves the undecidability of both trace inclusion for DOCA and universality for nondeterministic OCA. Jančar, Esparza, and Moller [9] consider trace inclusion between Petri Nets and finite systems and prove decidability in both directions. Jančar [8] showed that trace inclusion becomes undecidable if one compares processes of Petri Nets with at least two unbounded places. In [7], the authors show that trace inclusion is undecidable already for (nondeterministic) one-counter nets. Simulation preorder however, is known to be decidable and PSPACE-complete for this model $[1,10,6]$, which implies a PSPACE upper bound for trace inclusion on DOCN as trace inclusion and simulation coincide for deterministic systems.

Higuchi, Wakatsuki, and Tomita [5] compare the classes of languages defined by DOCN with various acceptance modes and in a series of papers consider the respective inclusion problems. They derive procedures that exhaustively search for a bounded witness that work in time and space polynomial in the size of the automata if the initial counter-values are fixed. We show that for monotone relations like trace inclusion or the inclusion of languages defined by acceptance with final states, one can speed up the search for suitable witnesses.

Our contribution. We fix the complexity of two well-known decidable decision problems regarding the traces of one-counter processes.

First, we show that trace inclusion between deterministic one-counter net is NL-complete. Our upper bound holds even if only the supposedly larger process is deterministic and if (binary encoded) initial counter-values are part of the input. This matches the trivial NL lower bound derived from DFA universality. Our technique uses short certificates for the existence of (possibly long) distinguishing traces. The sizes of certificates are polynomial in the number of states of the finite control and they can be verified in space logarithmic in the binary representation of the initial counter-values.

Our second result is that trace universality of nondeterministic OCN is Ackermann-complete. This problem can be easily seen to be (logspace) interreducible with checking trace inclusion between a finite process and a process of a OCN.

\section{Background}

We write $\mathbb{N}$ for the set of non-negative integers. For any set $A$, let $A^{*}$ denote the set of finite strings over $A$ and $\varepsilon \in A^{*}$ the empty string.

Definition 1 (One-Counter Nets). A one-counter net (OCN) is given as triple $\mathcal{N}=(Q$, Act,$\delta)$ where $Q$ is a finite set of control-states, Act is a finite set of action labels and $\delta \subseteq Q \times$ Act $\times\{-1,0,1\} \times Q$ is a set of transitions, each

written as $p \stackrel{a, d}{\longrightarrow} p^{\prime} . A$ process of $\mathcal{N}$ consists of a state $p \in Q$ and a counter-value $m \in \mathbb{N}$. We will simply write pm for such a pair. Processes can evolve according 
to the transition rules of the net: For any $a \in A c t, p, q \in Q$ and $m, n \in \mathbb{N}$ there is a step pm $\stackrel{a}{\longrightarrow}$ qn iff there exists $(p \stackrel{a, d}{\longrightarrow} q) \in \delta$ such that

$$
n=m+d \geq 0 .
$$

The net $\mathcal{N}$ is deterministic (a DOCN) if for every $p \in Q$ and $a \in$ Act, there is at most one transition $(p, a, d, q) \in \delta$. It is complete if for every $p \in Q$ and $a \in$ Act at least one transition $(p, a, d, q) \in \delta$ exists.

In this paper we will w.l.o.g. consider input nets in a certain normal form. Specifically, we assume what are sometimes called realtime automata, in which no silent ( $\varepsilon$-labelled) transitions are present. In the absence of zero-tests, the usual syntactic restriction for deterministic pushdown automata, that no state with outgoing $\varepsilon$-transition may have outgoing transitions labelled by $a \neq \varepsilon$ implies that all states on $\varepsilon$-cycles are essentially deadlocks and one can eliminate $\varepsilon$-labelled transitions in logarithmic space.

Definition 2 (Traces). Let pm be a process of the OCN $\mathcal{N}$. The traces of pm are the elements of the set

$$
T_{\mathcal{N}}(p m)=\left\{a_{1} a_{2} \ldots a_{k} \in A c t^{*} \mid \exists q n \text { pm } \stackrel{a_{0}}{\longrightarrow} \circ \stackrel{a_{1}}{\longrightarrow} \circ \cdots \circ \stackrel{a_{k}}{\longrightarrow} q n\right\} .
$$

We will omit the index $\mathcal{N}$ if is clear from the context. Trace inclusion is the decision problem that asks if $T_{\mathcal{A}}(\mathrm{pm}) \subseteq T_{\mathcal{B}}\left(p^{\prime} m^{\prime}\right)$ holds for given processes $\mathrm{pm}$ and $p^{\prime} m^{\prime}$ of nets $\mathcal{A}$ and $\mathcal{B}$, respectively. Trace universality asks if $A c t^{*} \subseteq T(\alpha)$ holds for a given process pm.

An important property of one-counter nets is that the step relation and therefore also trace inclusion is monotone with respect to the counter:

Lemma 1 (Monotonicity). If $p m \stackrel{a}{\longrightarrow} p^{\prime} m^{\prime}$ then $p(m+1) \stackrel{a}{\longrightarrow} p^{\prime}\left(m^{\prime}+1\right)$. This in particular means that $T(p m) \subseteq T(p(m+1))$ holds for any OCN-process $p m$.

The next lemma justifies our focus on processes of complete OCN. The proof is a simple construction and can be found in Appendix A. The idea is to first determinize $\mathcal{A}$ by consistently relabelling all transitions of $\mathcal{A}$ and $\mathcal{B}$, and then complete the net $\mathcal{B}$ by introducing a sink state.

Lemma 2 (Normal Form Assumption). Trace inclusion for OCN is logspacereducible to trace inclusion between a determinisic and a complete OCN. More precisely, given $O C N s \mathcal{A}$ and $\mathcal{B}$ with state sets $N$ and $M$, one can construct a $D O C N \mathcal{A}^{\prime}$ with states $N$ and a complete $O C N \mathcal{B}^{\prime}$ with states $M^{\prime} \supseteq M$ such that the following holds for any two processes pm and qn of $\mathcal{A}$ and $\mathcal{B}$, respectively:

$$
T_{\mathcal{A}}(p m) \subseteq T_{\mathcal{B}}(q n) \Longleftrightarrow T_{\mathcal{A}^{\prime}}(p m) \subseteq T_{\mathcal{B}^{\prime}}(q n) .
$$

Moreover, the constructed net $\mathcal{B}^{\prime}$ is deterministic if the original net $\mathcal{B}$ is.

Due to the undecidability of trace inclusion for OCN [7], a direct consequence of Lemma 2 is that trace inclusion $T_{\mathcal{A}}(p m) \subseteq T_{\mathcal{B}}(q n)$ is already undecidable if we allow the net $\mathcal{B}$ to be nondeterministic. Unless otherwise stated, we will from now on assume a DOCN $\mathcal{A}=\left(Q_{A}, A c t, \delta_{A}\right)$ and a complete DOCN $\mathcal{B}=$ $\left(Q_{B}, A c t, \delta_{B}\right)$. 


\section{Trace Inclusion for Deterministic One-Counter Nets}

We characterize witnesses for non-inclusion $T_{\mathcal{A}}(p m) \nsubseteq T_{\mathcal{B}}(q n)$, starting with some notation to express paths and their effects.

Definition 3 (OCN Paths). Consider the $O C N \mathcal{N}=(Q$, Act, $\delta)$. For the transition $t=\left(p, a, d, p^{\prime}\right) \in \delta$ we write source $(t)=p$, target $(t)=p^{\prime}$ and $\Delta(t)=d$ for its source and target states and counter-effect, respectively. A path in $\mathcal{N}$ is a sequence $\pi=t_{0} t_{1} \ldots t_{k} \in \delta^{*}$ of transitions where target $\left(t_{i}\right)=\operatorname{source}\left(t_{i+1}\right)$ for every $i<k$. Let ${ }^{i} \pi$ denote its prefix of length $i$. The effect $\Delta(\pi)$ and guard $\Gamma(\pi)$ of $\pi$ are

$$
\Delta(\pi)=\sum_{i=0}^{k} \Delta\left(t_{i}\right) \quad \text { and } \quad \Gamma(\pi)=-\min \left\{\Delta\left({ }^{i} \pi\right) \mid 0 \leq i \leq k\right\} .
$$

The path $\pi$ is enabled in process pm (write pm $\stackrel{\pi}{\longrightarrow}$ ) if $\Gamma(\pi) \leq m$. The source and target nodes of $\pi$ are those of its first and last transition, respectively. We write $p m \stackrel{\pi}{\longrightarrow} p^{\prime} m^{\prime}$ if $\pi$ takes $p m$ to $p^{\prime} m^{\prime}$, i.e., if $p m \stackrel{\pi}{\longrightarrow}$, target $(\pi)=p^{\prime}$ and $m^{\prime}=m+\Delta(\pi)$.

The guard $\Gamma(\pi)$ is the minimal counter-value that is sufficient to traverse the path $\pi$ while maintaining a non-negative counter-value along the way. This value is always non-negative. Notice that the absolute values of the effect and guard of a path are bounded by its length. We consider the synchronous product of the control graphs of two given deterministic one-counter nets.

Definition 4 (Product Paths). The product of nets $\mathcal{A}$ and $\mathcal{B}$ is the finite graph with nodes $V=Q_{\mathcal{A}} \times Q_{\mathcal{B}}$ and $($ Act $\times\{-1,0,1\} \times\{-1,0,1\})$-labelled edges $E$, where

$$
(p, q) \stackrel{a, d_{\mathcal{A}}, d_{\mathcal{B}}}{\longrightarrow}\left(p^{\prime}, q^{\prime}\right) \in E \text { iff } p \stackrel{a, d_{\mathcal{A}}}{\longrightarrow} p^{\prime} \in \delta_{\mathcal{A}} \text { and } q \stackrel{a, d_{\mathcal{B}}}{\longrightarrow} q^{\prime} \in \delta_{\mathcal{B}} .
$$

$A$ path in the product is a sequence $\pi=T_{0} T_{1} \ldots T_{k} \in E^{*}$ and defines paths $\pi_{\mathcal{A}}$ and $\pi_{\mathcal{B}}$ in nets $\mathcal{A}$ and $\mathcal{B}$, respectively. It is enabled in $(p m, q n)$ if $\pi_{\mathcal{A}}$ and $\pi_{\mathcal{B}}$ are enabled in pm and $q n$, respectively. In this case we write $(p m, q n) \stackrel{\pi}{\longrightarrow}\left(p^{\prime} m^{\prime}, q^{\prime} n^{\prime}\right)$ to mean that $p m \stackrel{\pi_{\mathcal{A}}}{\longrightarrow} p^{\prime} m^{\prime}$ and $q n \stackrel{\pi_{\mathcal{B}}}{\longrightarrow} q^{\prime} n^{\prime}$. We lift the definitions of source and target nodes to paths in the product: $\operatorname{source}(\pi)=\left(\operatorname{source}\left(\pi_{A}\right), \operatorname{source}\left(\pi_{B}\right)\right) \in$ $V, \operatorname{target}(\pi)=\left(\operatorname{target}\left(\pi_{A}\right), \operatorname{target}\left(\pi_{B}\right)\right) \in V$. Moreover, write $\Delta_{A}(\pi), \Delta_{B}(\pi)$, $\Gamma_{A}(\pi)$ and $\Gamma_{B}(\pi)$ for the effects and guards of $\pi$ in nets $\mathcal{A}$ and $\mathcal{B}$, respectively.

Since both $\mathcal{A}$ and $\mathcal{B}$ are deterministic and $\mathcal{B}$ is complete, a trace $w \in T_{\mathcal{A}}(p m)$ uniquely determines a path from state $(p, q)$ in their product. We therefore identify witnesses for non-inclusion with the paths they induce in the product.

Definition 5 (Witnesses). Assume $T_{\mathcal{A}}(p m) \nsubseteq T_{\mathcal{B}}(q n)$ for processes pm and qn of $\mathcal{A}$ and $\mathcal{B}$. $A$ witness for $(p m, q n)$ is a path $\pi$ in the product of $\mathcal{A}$ and $\mathcal{B}$ such that $(p m, q n) \stackrel{\pi}{\longrightarrow}\left(p^{\prime} m^{\prime}, q^{\prime} n^{\prime}\right)$ and for some $a \in A c t, p^{\prime} m^{\prime} \stackrel{a}{\longrightarrow}$ but $q^{\prime} n^{\prime} \stackrel{q}{\longrightarrow}$. 
Every witness $\pi$ for $(p m, q n)$ completely exhausts the counter in the process of $\mathcal{B}:(p m, q n) \stackrel{\pi}{\longrightarrow}\left(p^{\prime} m^{\prime}, q^{\prime} 0\right)$. This is because a process of a complete net can only not make an $a$-step in case the counter is empty.

Example 1. Consider two nets given by self-loops $p \stackrel{a, 0}{\longrightarrow} p$ and $q \stackrel{a,-1}{\longrightarrow} q$, respectively. Their product is the cycle $L=(p, q) \stackrel{a, 0,-1}{\longrightarrow}(p, q)$ with effects $\Delta_{A}(L)=$ 0 and $\Delta_{B}(L)=-1$. The only witness for $(p m, q n)$ for initial counter-values $m, n \in \mathbb{N}$ is $L^{n}$, which has length polynomial in the sizes of the nets and the initial counter-values, but not in the sizes of the nets alone.

The previous example shows that if binary-encoded initial counter-values are part of the input, we can only bound the length of shortest witnesses exponentially. However, we will see that it suffices to consider witnesses of a certain regular form only. This leads to small certificates for non-inclusion, which can be stepwise guessed and verified in space logarithmic in the size of the nets.

A crucial ingredient for our characterization is the monotonicity of witnesses, a direct consequence of the monotonicity of the steps in OCNs (Lemma 1):

Lemma 3. If $\pi$ is a witness for $(p m, q n)$ then for all $m^{\prime} \geq m$ and $n^{\prime} \leq n$ some prefix of $\pi$ is a witness for $\left(p m^{\prime}, q n^{\prime}\right)$.

The intuition behind the further characterization of witnesses is that in order to show non-inclusion, one looks for a path that is enabled in the process of $\mathcal{A}$ and moreover exhausts the counter in the process of $\mathcal{B}$. Since any sufficiently long path will revisit control-states in the product, we can compare such paths with respect to their effect on the counters and see that some are "better" than others. For instance, a cycle that only increments the counter in $\mathcal{B}$ and decrements the one in $\mathcal{A}$ is surely suboptimal considering our goal to find a (shortest) witness. The characterization Theorem 1 essentially states that if a witness exists, then also one that, apart from short paths, combines only the most productive cycles.

Definition 6 (Loops). A non-empty path $\pi$ in the product is called a cycle if $\operatorname{source}(\pi)=\operatorname{target}(\pi)$. Such a cycle is a loop if none of its proper subpaths is a cycle. The slope of loop $\pi$ is the ratio $S(\pi)=\Delta_{\mathcal{A}}(\pi) / \Delta_{\mathcal{B}}(\pi)$, where for $n>0$ and $k \in \mathbb{Z}$ we let $n / 0=\infty>k, 0 / 0=0$ and $-n / 0=-\infty<k$. Based on the effect of a loop we distinguish four types of loops: $(<,<),(>, \geq),(\leq, \geq)$, and $(\geq,<)$. The type of $\pi$ is Type $(\pi)=(\triangleleft, \bullet)$ iff $\Delta_{\mathcal{A}}(\pi) \triangleleft 0$ and $\Delta_{\mathcal{B}}(\pi) \triangleright 0$.

Note that no loop is longer than $|V|$ because it visits exactly one node twice.

Example 2. Consider two DOCN such that their product is the graph depicted below, where we identify transitions with their action labels for simplicity and 
let $v_{0}=\left(p, p^{\prime}\right) \in V$. The paths $t_{0} t_{1} t_{2}, t_{3} t_{4}$ and $t_{6}$ are loops with slopes $3 / 1,2 / 1$ and $1 / 1$ and types $(>, \geq),(>, \geq)$ and $(<,<)$, respectively. The path $\left(t_{0} t_{1} t_{2}\right)\left(t_{3} t_{4}\right)^{9} t_{5}\left(t_{6}\right)^{20}$ is a witness for $\left(p 0, p^{\prime} 10\right)$ of length 42 . By replacing 8 occurrences of the loop $\left(t_{3} t_{4}\right)$ with $\left(t_{0} t_{1} t_{2}\right)^{8}$ we derive the longer witness $\left(t_{0} t_{1} t_{2}\right)^{9}\left(t_{3} t_{4}\right) t_{5}\left(t_{6}\right)^{20}$, which has essentially the same structure but is more efficient in

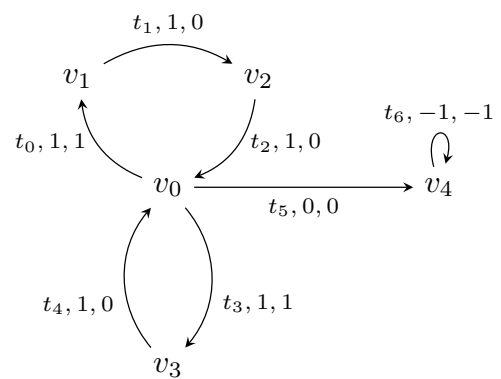
the sense that for the same effect on $\mathcal{B}$ it achieves a higher counter-effect on $\mathcal{A}$.

Theorem 1. Fix a $D O C N \mathcal{A}$, a complete $D O C N \mathcal{B}$, and let $K \in \mathbb{N}$ be the number of nodes in their product. There is a bound $c \in \mathbb{N}$ that depends polynomially on $K$, such that the following holds for any two processes pm and qn of $\mathcal{A}$ and $\mathcal{B}$. If $T(p m) \nsubseteq T(q n)$, then there is a witness for (pm,qn) that is either no longer than $c$ or has one of the following forms:

1. $\pi_{0} L_{0}^{l_{0}} \pi_{1}$, where $L_{0}$ is a loop of type $(\geq,<)$ and $\pi_{0}, \pi_{1}$ are no longer than $c$,

2. $\pi_{0} L_{0}^{l_{0}} \pi_{1} L_{1}^{l_{1}} \pi_{2}$, where $L_{0}$ and $L_{1}$ are loops of type $(>, \geq)$ and $(<,<)$ with $S\left(L_{0}\right)>S\left(L_{1}\right)$ and $\pi_{0}, \pi_{1}, \pi_{2}$ are no longer than $c$,

3. $\pi_{0} L_{0}^{l_{0}} \pi_{1}$, where $L_{0}$ is a loop of type $(<,<)$ and $\pi_{0}, \pi_{1}$ are no longer than $c$,

where in all cases, the number of iterations $l_{0}, l_{1} \in \mathbb{N}$ are polynomial in $K$ and the initial counter-values $m$ and $n$ of the given processes.

Proof (sketch). The overall idea of the proof is to explicitly rewrite witnesses into one of the canonical forms. More specifically, we introduce a system of path-rewriting rules which simplify witnesses by removing, reducing or changing some loops as in Example 2. We show that the rules preserve witnesses and any sequence of successive rule applications must eventually terminate with a normalized path, to which none of the rules is applicable. Such a witness can be decomposed as

$$
\pi=\pi_{0} L_{0}^{l_{0}} \pi_{1} L_{1}^{l_{1}} \ldots \pi_{k} L_{k}^{l_{k}} \pi_{k+1}
$$

where the $L_{i}$ are (pairwise different) loops and the $\pi_{i}$ are short, i.e. polynomially bounded. Moreover the rules are designed in such a way that almost all $l_{i}$ are polynomially bounded. By almost all we mean except one in the first and third form of the witness or two in the witness of the second form. This means that unravelling of those loops with polynomially bounded $l_{i}$ and glueing them with surrounding $\pi_{i}$ to get paths $\pi_{0}, \pi_{1}, \pi_{2}$ does not blow up of the length of $\pi_{0}, \pi_{1}, \pi_{2}$ above polynomial bound $c$.

Notice that the bound $c$ in the claim of Theorem 1 depends only on the number of states. We now derive a decision procedure for trace inclusion that works in logarithmic space.

Theorem 2. Let pm and qn be processes of $O C N \mathcal{A}$ and DOCN $\mathcal{B}$, respectively, where $m, n$ are given in binary. There is a nondeterministic algorithm that decides $T(p m) \subseteq T(q n)$ in logarithmic space. 
Proof. Let $\mathcal{A}=\left(Q_{\mathcal{A}}, A c t, \delta_{\mathcal{A}}\right)$ and $\mathcal{B}=\left(Q_{\mathcal{B}}, A c t, \delta_{\mathcal{B}}\right)$, and let $K \in \mathbb{N}$ be the number of states in their product. By Lemma 2, we can assume w.l.o.g. that $\mathcal{A}$ is deterministic and $\mathcal{B}$ is complete and deterministic and so Theorem 1 applies.

If the initial counter-values are $m=n=0$, Theorem 1 implies a polynomial bound on the length of shortest witnesses. In that case, one can simply stepwise guess and verify a witness, explicitly storing the intermediate processes with binary encoded counter-values in logarithmic space. Such a procedure is impossible with arbitrary initial counter-values as part of the input, because one does not even have the space to memorize them.

For the general case, we argue that one can nondeterministically guess a template (consisting of short paths) and verify in logspace that there is indeed some witness that fits this template. Theorem 1 allows us to either guess a short $(\leq c \in \operatorname{poly}(K))$ witness or one of forms 1,2 or 3 , together with matching short paths $\pi_{i}, L_{i}$. The effect and guard of these paths are bounded by their lengths and hence by $c$. This means $\mathcal{O}(\log K)$ space suffices to stepwise compute the binary representation of these values and verify that the conditions the form imposes on the types and slopes of the loops are met. It remains to check if exponents $l_{0}, l_{1} \in \mathbb{N}$ exist, that complete the description of a witness $\pi$. To see why these checks can be implemented in logarithmic space, first recall that one can verify inequalities of the form

$$
m \cdot A+B \geq n \cdot C+D
$$

in $\mathcal{O}(\log (A+B+C+D))$ space, if $m, n \in \mathbb{N}$ are given in binary (see Appendix B).

For templates of the first two forms, it suffices to check if $m \geq \Gamma_{\mathcal{A}}\left(\pi_{0} L_{0}\right)$, because the type of $L_{0}$ implies that $\Gamma_{\mathcal{A}}\left(\pi_{0} L_{0}^{l}\right) \leq \Gamma_{\mathcal{A}}\left(\pi_{0} L_{0}\right)$ for all $1<l \in \mathbb{N}$. This means that the process $p m$ of $\mathcal{A}$ can go to, and repeat the loop $L_{0}$ arbitrarily often. In case its effect in $\mathcal{B}$ is negative (in templates of form 1 ), this immediately implies the existence of a suitable $l_{0}$. For templates of form 2) the existence of $l_{0}, l_{1} \in \mathbb{N}$ completing the description of a witness is guaranteed because the slope of the first loop is bigger than that of the second.

For templates of the third kind recall that, because $\mathcal{B}$ is complete, a path $\pi=\pi_{0} L_{0}^{l_{0}} \pi_{1}$ is a witness iff there is some edge $T$ in the product such that $\Delta_{\mathcal{B}}(T)=-1$ and both $m \geq \Gamma_{\mathcal{A}}(\pi T)$ and $n+\Delta_{\mathcal{B}}(\pi T)=-1$. Equivalently, we can write this as

$$
\begin{aligned}
m+\Delta_{\mathcal{A}}\left(\pi_{0} L_{0}^{l_{0}}\right) & =m+\Delta_{\mathcal{A}}\left(\pi_{0}\right)+\Delta_{\mathcal{A}}\left(L_{0}\right) \cdot l_{0} \geq \Gamma_{\mathcal{A}}\left(\pi_{1} T\right) \text { and } \\
n+1 & =-\Delta_{\mathcal{B}}(\pi T)=-\Delta_{\mathcal{B}}\left(\pi_{0}\right)-\Delta_{\mathcal{B}}\left(L_{0}\right) \cdot l_{0}-\Delta_{\mathcal{B}}\left(\pi_{1} T\right) .
\end{aligned}
$$

Eliminating $l_{0}$, we see that this is true iff

$$
m+\Delta_{\mathcal{A}}\left(\pi_{0}\right)+\Delta_{\mathcal{A}}\left(L_{0}\right) \cdot \frac{\Delta_{\mathcal{B}}\left(\pi_{0}\right)+\Delta_{\mathcal{B}}\left(\pi_{1}\right)+n}{-\Delta_{\mathcal{B}}\left(L_{0}\right)} \geq \Gamma_{\mathcal{A}}\left(\pi_{1}\right) .
$$

Simplifying further we can bring this into the form $m \cdot A-n \cdot B \geq C$ where $A, B, C$ are polynomial in $c$. The condition can be checked in $\mathcal{O}(\log K)$ space. 


\section{Universality of Nondeterministic One-Counter Nets}

To contrast the result of the previous section we now turn to the problem of checking trace inclusion between a finite process and a nondeterministic OCN. This problem is known to be decidable, even for general Petri nets [9] and it can be easily seen to be (logspace) inter-reducible with the trace universality problem, because OCNs are closed under products with finite systems.

For OCN, trace universality can be decided using a simple well-quasi-order based saturation method that determinizes the net on the fly. We will see that this procedure is optimal: The problem is Ackermannian, i.e. it is non-primitive recursive and lies exactly at level $\omega$ of the Fast Growing Hierarchy [4].

Let $\mathbb{N}_{\perp}$ be the set of non-negative integers plus a special least element $\perp$ and let max be the total function that returns the maximal element of any nonempty finite subset and $\perp$ otherwise. Consider a set $S \subseteq Q \times \mathbb{N}$ of processes of an OCN $\mathcal{N}=(Q, A c t, \delta)$. We lift the definition of traces to sets of processes in the natural way: the traces of $S$ are $T(S)=\bigcup_{q n \in S} T(q n)$. By the monotonicity of trace inclusion (Lemma 1), the traces of a finite set of processes are determined only by the traces of its maximal elements.

Definition 7. Let $Q=\left\{q_{1}, q_{2}, \ldots, q_{k}\right\}$ be the states-set of some OCN. For a finite set $S \subseteq Q \times \mathbb{N}$ define the macrostate as the vector $M_{S} \in \mathbb{N}_{\perp}^{k}$ where for each $0<i \leq k, M_{S}(i)=M_{S}\left(q_{i}\right)=\max \left\{n \mid q_{i} n \in S\right\}$. In particular, the macrostate for a singleton set $S=\left\{q_{i} n\right\}$ is the vector with value $n$ at the $i$-th coordinate and $\perp$ on all others. The norm of a macrostate $M \in \mathbb{N}_{\perp}^{k}$ is $|M|_{\infty}=\max \{M(i) \mid 0<i \leq k\}$. We define a step relation $\stackrel{a}{\Longrightarrow}$ for all $a \in$ Act on the set of macrostates as follows:

$$
\left(n_{1}, n_{2}, \ldots, n_{k}\right) \stackrel{a}{\Longrightarrow}\left(m_{1}, m_{2}, \ldots, m_{k}\right)
$$

iff $m_{i}=\max \left\{n \mid \exists n_{j} \neq \perp . q_{j} n_{j} \stackrel{a}{\longrightarrow} q_{i} n\right\}$ for all $0<i \leq k$. The traces of macrostate $M$ are $T(M)=\bigcup_{0<i \leq k} T\left(q_{i} M(i)\right)$, where $T(q \perp)=\emptyset$. For two macrostates $M, N$ we say $M$ is covered by $N$ and write $M \sqsubseteq N$, if it is pointwise smaller, i.e., $M(i) \leq N(i)$ for all $0<i \leq k$. For convenience, we will write $\left\{q_{1}=n_{1}, q_{2}=n_{2}, \ldots, q_{l}=n_{l}\right\}$ for the macrostate with value $M(i)=n_{i}$ whenever $q_{i}=n_{i}$ is listed and $\perp$ otherwise.

Steps on macrostates correspond to the classical powerset construction and each macrostate represents the finite set of possible processes the OCN can be in, where all non-maximal ones (w.r.t. their counter-value) are pruned out.

Example 3. Macrostate 
Consider automaton $\mathcal{A}$ like on the picture, state $q_{3}$ and a counter value 4 ; we analyse traces, $T\left(q_{3} 4\right)$. If we go via an edges labelled by $a$ once we can see that $T\left(q_{3} 4\right)=\{\varepsilon\} \cup a T\left(q_{2} 3\right) \cup$ $a T\left(q_{3} 5\right)$. This implies that $T\left(q_{3} 4\right)$ is univer$a, 1 \overbrace{q 2}^{q 1} q 3 \supset a,-1$ sal iff $T\left(q_{3}, 5\right) \cup T\left(q_{2}, 3\right)$ is universal, i.e. contains $A c t^{*}$. Making similar analysis after using two more $a$ we get that $T\left(q_{3} 4\right)$ is universal iff $T\left(q_{3} 7\right) \cup T\left(q_{2} 5\right) \cup T\left(q_{1} 5\right) \cup T\left(q_{3} 4\right)$ is universal. But we know that $T\left(q_{3} 4\right) \subseteq T\left(q_{3} 7\right)$ which implies that $T\left(q_{3} 7\right) \cup T\left(q_{2} 5\right) \cup T\left(q_{1} 5\right) \cup T\left(q_{3} 4\right)=$ $T\left(q_{3} 7\right) \cup T\left(q_{2} 5\right) \cup T\left(q_{1} 5\right)$. This immediately lead to introduce macrostates $M_{\left\{q_{3} 7, q_{2} 5, q_{1} 5, q_{3} 4\right\}}=$ $(5,5,7)$. The norm $\left|M_{\left.\left\{q_{3} 7, q_{2} 5, q_{1} 5, q_{3}\right\}\right\}}\right|_{\infty}=7$. On the other hand $M_{\left\{q_{3} 4\right\}}=(\perp, \perp, 4)$ which means that states $q_{1}$ and $q_{2}$ are not present and in this case $M(1)=M\left(q_{1}\right)=\perp$. Moreover we can write that $M_{\left\{q_{3} 4\right\}} \sqsubseteq M_{\left\{q_{3} 7, q_{2} 5, q_{1} 5, q_{3} 4\right\}}$.

The next lemma directly follows from these definitions and monotonicity (Lemma 1).

\section{Lemma 4.}

1. The covering-order $\sqsubseteq$ is a well-quasi-order on $\mathbb{N}_{\perp}^{k}$, the set of all macrostates. Moreover, $M \sqsubseteq N$ implies $T(M) \subseteq T(N)$.

2. If $M \stackrel{a}{\Longrightarrow} N$ then $|N|_{\infty} \leq|M|_{\infty}+1$.

3. For any finite set $S \subseteq Q \times \mathbb{N}$ it holds that $T(S)=T\left(M_{S}\right)$.

Dealing with macrostates allows us to treat universality as a reachability problem: By point 3 of Lemma 4 we see that a process $q n$ is not trace universal, $A c t^{*} \neq T(q n)$, if and only if $M_{\{q n\}} \Longrightarrow^{*}(\perp, \perp, \ldots, \perp)$. We take the perspective of a pathfinder, whose goal it is to reach $(\perp)^{k}$.

We can decide universality by stepwise guessing a shortest terminating path from the initial macrostate, and thus a witness for non-universality. Whenever we see a macrostate that covers one of its predecessors, we can safely discard this candidate, because omitting the intermediate path would result in a shorter witness by Lemma 4.1 .

We show non-primitive recursiveness by reduction from the control-state reachability problem for incrementing counter machines [3, 4].

Definition 8 (Counter machines). A (Minsky)-counter machine (CM) is an automaton with finitely many states $Q$, finitely many counters $C_{1}, C_{2}, \ldots, C_{k}$, and transitions are of the form $Q \times$ Act $\times Q$ where Act is \{inc, dec,ifz $\times$ $\{1,2, \ldots, k\}$. A configuration of such a $C M$ consists of a state and a valuation of the counters. Performing a transition $(p,(o p, i), q)$ changes a configuration precisely: the state changes from $p$ to $q$ and we make operation op on the counter $c_{i}$, where inc, dec and ifz mean increment, decrement and zero-test, respectively. 
Such a step is forbidden if the requested operation is dec and the value of $c_{i}$ is 0 , or if $c_{i}>0$ and the operation is ifz.

An incrementing counter machine (ICM) is a CM in which counters can spontaneously increment without performing any transitions. Such increments we call incrementing errors. Control-state reachability is the decision problem that asks if there is a run of a given CM from an initial configuration to some given state $q_{f} \in Q$.

Our reduction is based on the following simple observation. Consider an OCN $\mathcal{N}=(Q$, Act,$\delta)$ that contains a universal state $U$ : it has self-loops $U \stackrel{a, 0}{\longrightarrow} U \in \delta$ for every action $a \in$ Act. A Pathfinder who wants to prove non-universality must avoid macrostates with $M(U) \neq \perp$, because no continuation of a path leading to such a macrostate can be a witness. We can use this idea to construct macrostates that prevent Pathfinder from making certain actions.

Definition 9 (Obstacles). Let $S \subseteq$ Act be a set of actions in an OCN that contains a universal state $U$. A state $q \in Q$ is called an S-obstacle if $q \stackrel{a, 0}{\longrightarrow} U \in \delta$ for all actions $a \in S$. We say $q$ ignores $S$, if $q \stackrel{a, 0}{\longrightarrow} q \in \delta$ for all $a \in S$.

Note that if a macrostate contains an $S$-obstacle, then Pathfinder must avoid all actions of $S$. In order to remove an obstacle, Pathfinder must play an action that is not the label of any of its incoming transitions.

Theorem 3. Trace universality for $O C N$ is not primitive recursive.

Proof. By reduction from the control-state reachability problem for ICM, which has non-primitive recursive complexity [3]. We construct an OCN-process Init (0) that is not universal iff a given ICM reaches a final state from its initial configuration. The idea is to enforce a faithful simulation of the ICM by pathfinder, who wants to show non-universality of the OCN by stepwise rewriting the initial macrostate $\{$ Init $=0\}$ to the all-bottom-macrostate $\perp^{l}$.

We construct an OCN $\mathcal{N}$ which has a unique action for every transition of the ICM, as well as actions $\tau_{i}$ that indicate incrementing errors for every counter $c_{i}$, and actions $\sharp$ and $\$$ to mark the beginning and end of a run, respectively. This way we make sure there is a strict correspondence between words and ICM-runs. The states of $\mathcal{N}$ are

- a new initial state Init and a universal state $U$,

- a state $q_{i}$ for every state $q_{i}$ of the ICM,

- a state $C_{i}$ for every counter $c_{i}$ of the ICM,

- a state $Z$, which ignores every action but the end marker $\$$. State $Z$ will be used to access the constant 0 .

A configuration $q\left(c_{1}, c_{2}, \ldots, c_{k}\right)$ of the ICM is represented by a macrostate $\left\{q=0, Z=0, C_{1}=c_{1}, C_{2}=c_{2}, \ldots, C_{k}=c_{k}\right\}$. We will define the transitions of $\mathcal{N}$ such that the only way for Pathfinder to reach $\perp^{l}$ is by rewriting the initial macrostate $\{$ Init $=0\}$ to the one representing the initial ICM configuration and 
then to stepwise announce the transitions of an accepting run of the ICM. Using the idea of obstacles, we define the rules of the net $\mathcal{N}$ so that the only way Pathfinder can avoid the universal state $U$ and reach the macrostate $\perp^{k}$ is by first transforming the initial macrostate $\{$ Init $=0\}$ to the one that represents the initial ICM configuration and then announcing transitions (as well as actions demanding increment errors) of a valid and accepting run of the ICM.

Initialization. To set up $M_{0}=\left\{q_{0}=0, Z=0, C_{0}=0, C_{1}=0, \ldots, C_{k}=0\right\}$, representing the initial ICM configuration, we add \#-labelled transitions with effect 0 from Init to $q_{0}, Z$ and $C_{i}$ for all $0 \leq i \leq k$. Moreover, we make Init an obstacle for every action but $\sharp$. This way, Pathfinder has to play $\sharp$ as the first move (and set up $M_{0}$ ) in order to avoid a universal macrostate. Furthermore we make \# an obstacle for every state except of Init; this prevent playing \# after the first move.

Finite control. For any transition $t=q \stackrel{(a, i)}{\longrightarrow} q^{\prime}$ of the ICM, we add a transition $q \stackrel{t, 0}{\longrightarrow} q^{\prime}$ to $\mathcal{N}$ that, in a macrostate-step, will replace the value 0 in dimension $q$ by $\perp$ and introduce value 0 in dimension $q^{\prime}$. Moreover, we make every state $q$ an obstacle for all actions announcing ICM-transitions not originating in $q$. This prevents Pathfinder from announcing transitions from $q$ unless the current macrostate has $M(q)=0$ and $M\left(q_{i}\right)=\perp$ for all $q_{i} \neq q$.

Simulation of the Counters. Every transition operates on one of the counters $c_{i}$ for $0 \leq i \leq k$. Below we list the corresponding transitions in the OCN $\mathcal{N}$ for this counter. Every state of $\mathcal{N}$ not explicitly mentioned ignores the action in question. In the macrostate, the values of these states are therefore unchanged.

increments For ICM-transitions $t$ that increase the $i$ th counter, $\mathcal{N}$ contains a $t$-labelled transition from state $C_{i}$ to $C_{i}$ with effect +1 . Additionally, to deal with spontaneous increment errors, there is a $\tau_{i}$-labelled increasing self-loop in state $C_{i}$.

decrements For ICM-transitions $t$ that decrease the $i$ th counter, $\mathcal{N}$ contains a $t$-labelled transition from state $C_{i}$ to $C_{i}$ with effect -1 .

This means that the next macrostate $M$ could lose the value for this counter and have $M\left(C_{i}\right)=\perp$ if previously, the value was 0 . In that case, the decrementing step from value 0 to value 0 is valid in the ICM because it can first (silently) increment and then do the (visible) decrement step. In order to avoid losing the state $C_{i}$ in the macrostate, the OCN contains a transition $Z \stackrel{t, 0}{\longrightarrow} C_{i}$ from the constant-zero state $Z$ to state $C_{i}$. Recall that $Z$ is present in the macrostate because $Z$ ignores every action except the end marker $\$$. Consequently, no correctly set up macrostate will set $M\left(C_{i}\right)=\perp$.

zero-tests For ICM-transitions $t$ that test the $i$ th counter for 0 , we add a $t$ labelled transition $C_{i} \stackrel{t,-1}{\longrightarrow} U$ from state $C_{i}$ to the universal state. This prevents Pathfinder from using these actions if the current macrostate has 
$M\left(C_{i}\right)>0$ because it would make the next macrostate universal. If however $M\left(C_{i}\right)=0$, such a step is safe because the punishing transition is not enabled in the OCN-process $C_{i} 0$.

Lastly, we only add transitions to $\mathcal{N}$ so that the final state $q_{f}$ is the only original ICM-state which is not an obstacle for $\$$. This prevents Pathfinder from playing the end-marker $\$$ unless the simulation has reached the final state.

Example 4. Reduction.

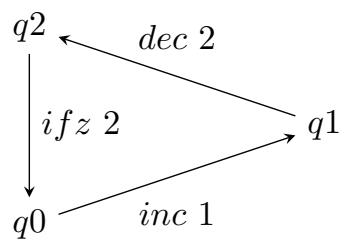

Consider an incrementing error two counter machine (as on the left) and we ask about reachability from $q_{0}$ to $q_{2}$.

The one counter net which is result of the construction for the above reachability problem. We will use several universal states $U$ to avoid crossing arrows, moreover edges labelled with a sequence of labels mean a bunch of edges one for each label. We put labels into brackets, to clearly separate each label.

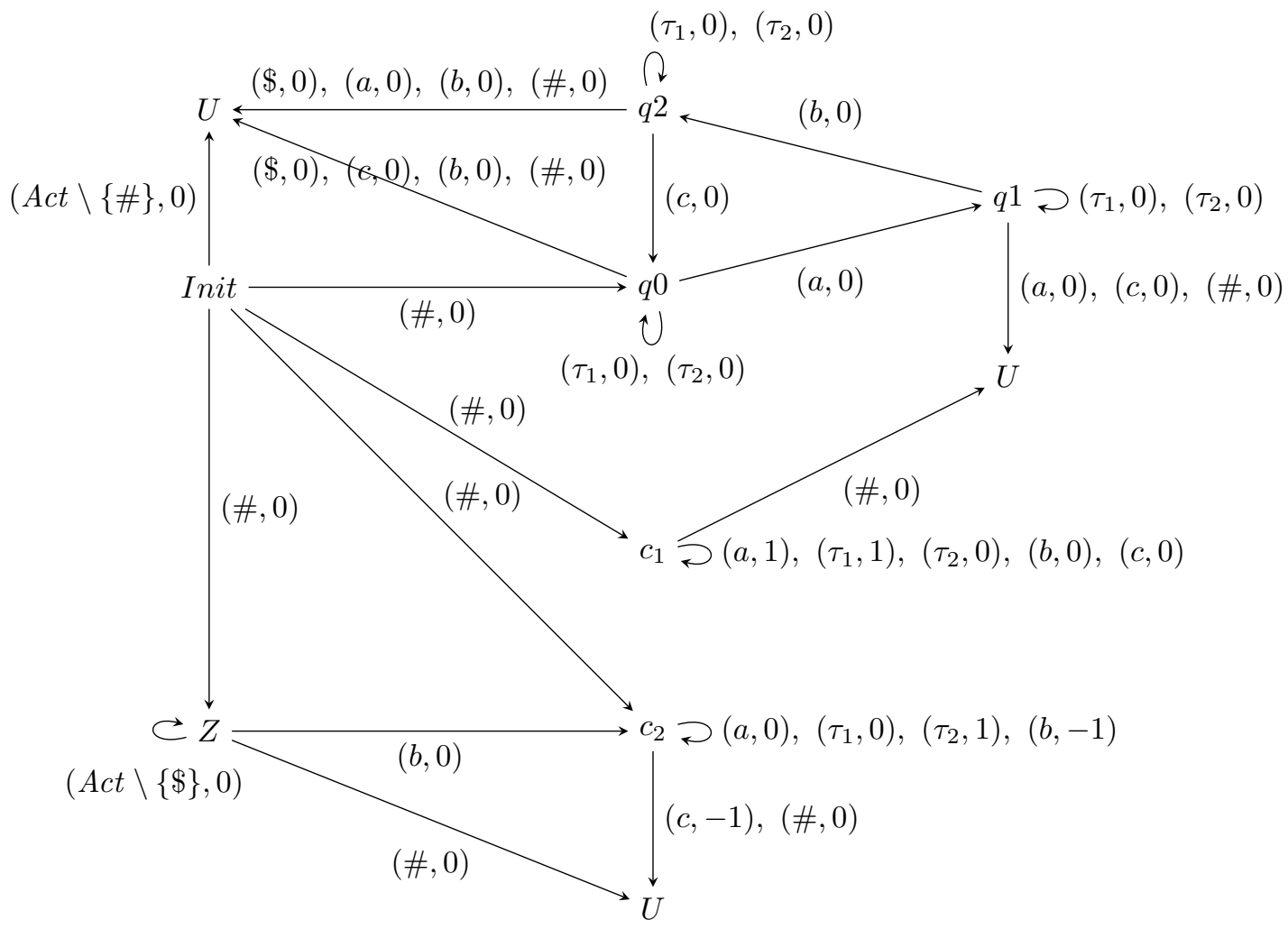


For the rest of this section, we recall a recent result from Figueira, Figueira, Schmitz, and Schnoebelen [4], that allows us to provide the exact complexity of the OCN trace universality problem in terms of its level in the Fast-Growing Hierarchy.

Definition 10 (Fast-Growing Hierarchy). Consider the family of functions $F_{n}: \mathbb{N} \rightarrow \mathbb{N}$ where for $x, k \in \mathbb{N}$,

$$
F_{0}(x)=x+1 \quad \text { and } \quad F_{k+1}(x)=F_{k}^{x+1}(x) .
$$

Here, $F^{k}$ denotes the $k$-fold application of $F$. Moreover, define $F_{\omega}(x)=F_{x}(x)$ for the first limit ordinal $\omega$. For $k \leq \omega, \mathfrak{F}_{k}$ denotes the least class of functions that contains all constants and is closed under substitution, sum, projections, limited recursion and applications of functions $F_{n}$ for $n \leq k$.

Already $\mathfrak{F}_{2}$ contains all elementary functions and the union $\bigcup_{k \in \mathbb{N}} \mathfrak{F}_{k}$ of all $f$ nite levels contains exactly the primitive-recursive functions. A function is called Ackermannian if it is in $\mathfrak{F}_{\omega} \backslash \bigcup_{k \in \mathbb{N}} \mathfrak{F}_{k}$.

A sequence $x_{0}, x_{1}, \ldots, x_{l}$ of macrostates is called good if there are indices $0 \leq i<j \leq l$ such that $x_{i} \sqsubseteq x_{j}$ and bad otherwise. The sequence is $t$-controlled by $f: \mathbb{N} \rightarrow \mathbb{N}$ if $\left|x_{i}\right|_{\infty}<f(i+t)$ for every index $0 \leq i \leq l$.

Theorem 4 ([4]). Let $f: \mathbb{N} \rightarrow \mathbb{N}$ be a monotone function in $\mathfrak{F}_{\gamma}$ such that $f(x) \geq \max \{1, x\}$ for some $\gamma \geq 1$. There is a function $L_{k, f}(t)$ in $\mathfrak{F}_{k+\gamma-1}$ that computes a bound on the maximal length of bad sequences in $\mathbb{N}_{\perp}^{k}$ that are $t$ controlled by $f$.

Corollary 1. Trace universality of $O C N$ is Ackermannian.

Proof. By Theorem 3, it suffices to show that the problem is in $\mathfrak{F}_{\omega}$. Recall the procedure that, for a given process $p m$ of a net with $k$ control-states, guesses a shortest terminating path from the initial macrostate (a witness for nonuniversality), and stops unsuccessfully if a macrostate covers one that has been seen before. The time and space requirements of this procedure are bounded in terms of the longest non-increasing (w.r.t. covering) sequence of $k$-dimensional macrostates. These are bad sequences where the norm of the initial macrostate is $m$, the counter-value of the process to check for universality. By point 2 of Lemma 4, such sequences are $m$-controlled by the successor function $f(x)=x+1$, which is in $\mathfrak{F}_{1}$. By Theorem 4, computing the bound and running the procedure above is in $\mathfrak{F}_{k}$. As $k$ is part of the input, this yields a procedure in $\mathfrak{F}_{\omega}$.

\section{Conclusion}

We have shown NL-completeness of the general trace inclusion problem for deterministic one-counter nets, where initial counter-values are part of the input. Our proof is based on a characterization of the shape of possible witnesses in terms of a small number of polynomially-sized templates. Realizability of such 
templates can be verified in space logarithmic only in the size of the underlying state space. Our procedure is therefore independent of the number of action symbols and transitions in the input nets. To prove the characterization theorem we use witness rewriting rules, the correctness of which crucially depends on the monotonicity of trace inclusion w.r.t. counter-values. In fact, we only make use of this property in the net on the left but similarly one can define rules that exploit only the monotonicity in the process on the right. With some additional effort one can extend this argument also for trace inclusion between DOCN and DOCA or vice versa (see [13]).

The second part of the paper explores the complexity of the universality problem for nondeterministic OCN, and trace inclusion between finite systems and OCN that easily reduces to OCN universality. Here we show that the simplest known algorithm which uses a well-quasi-order based saturation technique has already optimal complexity: The problem is Ackermannian, i.e., not primitive recursive.

Acknowledgement. We thank Mary Cryan, Diego Figueira and Sylvain Schmitz for helpful discussions and the anonymous reviewers of an earlier draft for their constructive feedback. Piotr Hofman acknowledges a partial support by the Polish NCN grant 2013/09/B/ST6/01575.

\section{References}

[1] A. Abdulla and K. Čerāns. "Simulation Is Decidable for One-Counter Nets". In: CONCUR. 1998, pp. 253-268.

[2] S. Böhm, S. Göller, and P. Jančar. "Equivalence of Deterministic OneCounter Automata is NL-complete". In: STOC. 2013, pp. 131-140.

[3] S. Demri and R. Lazić. "LTL with the freeze quantifier and register automata". In: ACM Trans. Comput. Logic 10.3 (Apr. 2009), 16:1-16:30.

[4] D. Figueira, S. Figueira, S. Schmitz, and P. Schnoebelen. "Ackermannian and Primitive-Recursive Bounds with Dickson's Lemma". In: LICS. 2011, pp. 269-278.

[5] K. Higuchi, M. Wakatsuki, and E. Tomita. "Some Properties of Deterministic Restricted One-Counter Automata". In: IEICE E79-D.8 (July 1996), pp. 914-924.

[6] P. Hofman, S. Lasota, R. Mayr, and P. Totzke. "Simulation Over Onecounter Nets is PSPACE-Complete". In: FSTTCS. 2013, pp. 515-526.

[7] P. Hofman, R. Mayr, and P. Totzke. "Decidability of Weak Simulation on One-Counter Nets". In: LICS. 2013, pp. 203-212.

[8] P. Jančar. "Undecidability of Bisimilarity for Petri Nets and Some Related Problems". In: TCS 148.2 (1995), pp. 281-301.

[9] P. Jančar, J. Esparza, and F. Moller. "Petri Nets and Regular Processes". In: J. Comput. Syst. Sci. 59.3 (1999), pp. 476-503.

[10] P. Jančar, A. Kučera, and F. Moller. "Simulation and Bisimulation over One-Counter Processes". In: STACS. 2000, pp. 334-345. 
[11] G. Sénizergues. "L(A) = L(B)?" In: ENTCS 9 (1997), p. 43.

[12] C. Stirling. "Deciding DPDA Equivalence Is Primitive Recursive". In: ICALP. 2002, pp. 821-832.

[13] P. Totzke. "Inclusion Problems for One-Counter Systems". PhD thesis. LFCS, University of Edinburgh, 2014.

[14] L. Valiant. "Decision Procedures for Families of Deterministic Pushdown Automata". PhD thesis. University of Warwick, 1973.

[15] L. Valiant and M. S. Paterson. "Deterministic One-Counter Automata". In: JCSS 10.3 (1975), pp. $340-350$. 


\section{A Normal-Form Assumption}

We consider here what is sometimes called realtime automata, in which no silent ( $\varepsilon$-labelled) transitions are present. In the absence of zero-tests, the usual syntactic restriction for deterministic Pushdown Automata, (no state with outgoing $\varepsilon$-transition may have outgoing transitions labelled by $a \neq \varepsilon$ ) and the lack of an explicit zero-test in our model implies that all states on $\varepsilon$-cycles are essentially deadlocks. A process in such a state can either silently exhaust the counter and deadlock or divert into an infinite $\varepsilon$ loop. With respect to their traces, those processes are equivalent. This means one can eliminate $\varepsilon$-transitions by removing $\varepsilon$-cycles and replacing the remaining short paths by direct steps (and normalize the effects of single transitions back to $\{-1,0,1\}$ ). Such a reduction works in $\mathcal{O}(\log n)$ space. Allowing $\varepsilon$-transitions thus leaves the complexity of trace inclusion invariant.

Lemma 2 (Normal Form Assumption). Trace inclusion for OCN is logspacereducible to trace inclusion between a determinisic and a complete OCN. More precisely, given $O C N s \mathcal{A}$ and $\mathcal{B}$ with state sets $N$ and $M$, one can construct a DOCN $\mathcal{A}^{\prime}$ with states $N$ and a complete $O C N \mathcal{B}^{\prime}$ with states $M^{\prime} \supseteq M$ such that the following holds for any two processes pm and qn of $\mathcal{A}$ and $\mathcal{B}$, respectively:

$$
T_{\mathcal{A}}(p m) \subseteq T_{\mathcal{B}}(q n) \Longleftrightarrow T_{\mathcal{A}^{\prime}}(p m) \subseteq T_{\mathcal{B}^{\prime}}(q n) .
$$

Moreover, the constructed net $\mathcal{B}^{\prime}$ is deterministic if the original net $\mathcal{B}$ is.

Proof. Let $\mathcal{A}=\left(N, A c t, \delta_{A}\right)$ and $\mathcal{B}=\left(M, A c t^{\prime}, \delta_{B}\right)$. If $\mathcal{A}$ is not already deterministic, we can make it so by uniquely re-labeling all its transitions $t$ by actions $a_{t}$ and adding corresponding transitions $\left(p^{\prime}, a_{t}, d^{\prime}, q^{\prime}\right)$ to the other net $\mathcal{B}$ for any existing $\left(p^{\prime}, a, d^{\prime}, q^{\prime}\right) \in \delta_{B}$, where $a$ is the original label of $t \in \delta_{A}$. So assume $\mathcal{A}$ is deterministic and pick a new action label $\$ \notin$ Act. We add $\$$-labelled cycles with effect 0 to all states of $\mathcal{A}$ : The new net $\mathcal{A}^{\prime}=\left(N, \operatorname{Act} \cup\{\$\}, \overline{\delta_{A}}\right)$ has transitions $\overline{\delta_{A}}=\delta_{A} \cup\{(s, \$, 0, s) \mid s \in N\}$. To compensate this, we add $\$$-cycles to all states of $\mathcal{B}$ in the same way. We add a sink state $L$ (for losing), which has counter-decreasing cycles for all actions, and connect all states without outgoing $a$-transitions to $L$ by $a$-labelled transitions. $\mathcal{B}^{\prime}=\left(M \cup\{L\}, A c t \cup\{\$\}, \overline{\delta_{B}}\right)$ where

$$
\begin{aligned}
\overline{\delta_{B}}=\delta_{B} & \cup\{(s, \$, 0, s) \mid s \in M\} \\
& \left.\cup\left\{(s, a, 0, L) \mid a \in \text { Act and } s \stackrel{a}{\longrightarrow} s^{\prime} \notin \delta \text { for any } s^{\prime} \in M\right)\right\} \\
& \cup\{(L, a,-1, L \mid a \in A c t \cup\{\$\}\} .
\end{aligned}
$$

We see that if a word $w$ of length $k$ witnesses non-inclusion $T_{\mathcal{A}}(q n) \nsubseteq T_{\mathcal{B}}\left(q^{\prime} n^{\prime}\right)$ then there is a word that witnesses non-inclusion $T_{\mathcal{A}^{\prime}}(q n) \nsubseteq T_{\mathcal{B}^{\prime}}\left(q^{\prime} n^{\prime}\right)$ To see this, observe that in this case, any $w$-labelled path in $\mathcal{B}^{\prime}$ that starts in state $q^{\prime}$ must end in state $L$. This means any such path takes the initial process $q^{\prime} n^{\prime}$ to some process $L n^{\prime \prime}$ where $n^{\prime \prime} \leq n^{\prime}+k$ and now by playing $n^{\prime \prime}$ times a label $\$$ we get a new witness. Conversely, if there is a witness $w$ for $T_{\mathcal{A}^{\prime}}(q n) \nsubseteq T_{\mathcal{B}^{\prime}}\left(q^{\prime} n^{\prime}\right)$ then the shortest such witness must be of the form $w=w^{\prime} \$^{k}$ where $w^{\prime}$ does not contain actions $\$$ because as $\$$-labelled steps leave any process not in state $L$ unchanged. This means $w^{\prime}$ witnesses $T_{\mathcal{A}}(q n) \nsubseteq T_{\mathcal{B}}\left(q^{\prime} n^{\prime}\right)$. 


\section{B Checking Weighted Inequalities in Logspace}

Lemma 5. Inequalities of the form $m \cdot A+B \geq n \cdot C+D$ where all coefficients are non-negative integers given in binary can be verified in $\mathcal{O}(\log (A+B+C+D))$ deterministic space.

Proof. Assume w.l.o.g. that the bit-representations of $m$ and $n$ are of the same length, as are those of $A, B, C$ and $D$, and we have the least significant bit on the right.

To check $m \geq n$, we can stepwise read their binary representation from right to left, flipping an "output" bit Out on the way: Initially, Out $:=1$; in every step set $O u t:=0$ if the current bit in $m$ is strictly smaller than that in $n$; set Out $:=1$ if the current bit in $m$ is strictly bigger than that in $n$ and otherwise proceed without touching $O u t$. The inequality holds iff $O u t=1$ after completely reading the input.

To check the weighted variant, we use the same algorithm but multiply $m$. $A$, and $n \cdot C$ on the fly, using standard long binary multiplication. We use a scratchpad to store the intermediate sums, starting with values $B$ and $D$. In a step that reads the $i$ th bit $m[i]$ of $m$, we want to add $A \cdot 2^{i}$ to the intermediate sum if $m[i]=1$. We can do that by shifting the binary representation of $A$ left $i$ times and adding the result to the current scratchpad. We see that none of the bits up to $i-1$ in the scratchpad are affected by this operation. We can therefore discard (and use for the comparison in our simple algorithm above) the rightmost bit of the scratchpad in every step. The claim now follows from the observation that the necessary size of the scratchpad is bounded by $B+A+1$. 


\section{Proof of Theorem 1}

We show that it is safe to consider only witnesses in a reduced form, and derive bounds on the length of certain subpaths. For this, we introduce path rewriting rules that exchange occurrences of some loops by others. We then show (in Lemma 6) that these rules preserve witnesses and (Lemma 7) cannot be applied indefinitely. For reduced witnesses, those to which no rules are applicable, we derive (Lemma 8) bounds on the multiplicities of loops that are less productive than others, which will enable us to prove Theorem 1.

For the rest of this section let $V$ and $E$ be the sets of nodes and edges in the product of $\mathcal{A}$ and $\mathcal{B}$.

We start with an easy observation: Because no loop $L$ is longer than $|V|$, we conclude that $\left(\Delta_{\mathcal{A}}(L), \Delta_{\mathcal{B}}(L)\right) \in\{-V \ldots V\} \times\{-V \ldots V\}$, so there are $F_{0}:=$ $(2 \cdot|V|+1)^{2}$ different values the pair $\Delta_{\mathcal{A}}(L), \Delta_{\mathcal{B}}(L)$ can have. Moreover, if a witness exists, then also one that does not contain different loops with the same effects: if $\pi_{0} L_{0} \pi_{1} L_{1} \pi_{2}$ is a witness where $\left|\pi_{1}\right|>0$ and $L_{0}, L_{1}$ are two loops with $\Delta\left(L_{0}\right)=\Delta\left(L_{1}\right)$, then either some prefix of $\pi_{0} L_{0}^{2} \pi_{1} \pi_{2}$ (if $\Delta_{A}\left(L_{0}\right) \geq 0$ ) or some prefix of $\pi_{0} \pi_{1} L_{1}^{2} \pi_{2}$ (if $\Delta_{A}\left(L_{0}\right)<0$ ) must also be a witness by Lemma 3. We can therefore consider w.l.o.g. only sane paths, which are of the form

$$
\pi=\pi_{0} L_{0}^{l_{0}} \pi_{1} L_{1}^{l_{1}} \ldots \pi_{r} L_{r}^{l_{r}} \pi_{r+1}
$$

where $r \leq F_{0}$, all $\pi_{i}$ are acyclic and all loops have pairwise different effects.

Definition 11 (Path Rewriting Rules). Consider the rules given below.

$$
\begin{aligned}
& \quad \boldsymbol{U} \boldsymbol{U L} \\
& \pi=\pi_{0} L_{0}^{l_{0}} \pi_{1} L_{1}^{l_{1}} \pi_{2} \\
& \text { Type }\left(L_{0}\right)=(>, \geq) \\
& \text { Type }\left(L_{1}\right)=(>, \geq) \\
& \Delta_{\mathcal{B}}\left(L_{0}\right) \cdot x=\Delta_{\mathcal{B}}\left(L_{1}\right) \cdot y \\
& S\left(L_{0}\right) \geq S\left(L_{1}\right) \\
& \underline{l_{1}-y>0} \\
& \rho=\pi_{0} L_{0}^{l_{0}+x} \pi_{1} L_{1}^{l_{1}-y} \pi_{2}
\end{aligned}
$$$$
\text { UUR }
$$$$
\pi=\pi_{0} L_{0}^{l_{0}} \pi_{1} L_{1}^{l_{1}} \pi_{2}
$$$$
\operatorname{Type}\left(L_{0}\right)=(>, \geq)
$$$$
\text { Type }\left(L_{1}\right)=(>, \geq)
$$$$
\Delta_{\mathcal{B}}\left(L_{0}\right) \cdot x=\Delta_{\mathcal{B}}\left(L_{1}\right) \cdot y
$$$$
S\left(L_{0}\right)<S\left(L_{1}\right)
$$$$
\frac{l_{0}-x>\left|\pi_{1} L_{1}\right|}{\rho=\pi_{0} L_{0}^{l_{0}-x} \pi_{1} L_{1}^{l_{1}+y} \pi_{2}}
$$

\section{$U D$}

$\pi=\pi_{0} L_{0}^{l_{0}} \pi_{1} L_{1}^{l_{1}} \pi_{2}$

$\operatorname{Type}\left(L_{0}\right)=(>, \geq)$

Type $\left(L_{1}\right)=(<,<)$

$\Delta_{\mathcal{B}}\left(L_{0}\right) \cdot x=-\Delta_{\mathcal{B}}\left(L_{1}\right) \cdot y$

$S\left(L_{0}\right) \leq S\left(L_{1}\right)$

$l_{0}-x \geq\left|\pi_{1}\right|$

$\underline{l_{1}-y>0 \wedge l_{0}-x>0}$

$\rho=\pi_{0} L_{0}^{l_{0}-x} \pi_{1} L_{1}^{l_{1}-y} \pi_{2}$

$$
\begin{aligned}
& \pi=\pi_{0} L_{0}^{l_{0}} \pi_{1} L_{1}^{l_{1}} \pi_{2} \\
& \text { Type }\left(L_{0}\right)=(<,<) \\
& \text { Type }\left(L_{1}\right)=(<,<) \\
& \Delta_{\mathcal{B}}\left(L_{0}\right) \cdot x=\Delta_{\mathcal{B}}\left(L_{1}\right) \cdot y \\
& S\left(L_{0}\right)<S\left(L_{1}\right) \\
& l_{1}>\left|L_{0}\right| \cdot x+2\left|\pi_{1}\right| \\
& \underline{l_{1}-y>0} \\
& \rho=\pi_{0} L_{0}^{l_{0}+x} \pi_{1} L_{1}^{l_{1}-y} \pi_{2}
\end{aligned}
$$

$$
\begin{aligned}
& \quad \boldsymbol{D D R} \\
& \pi=\pi_{0} L_{0}^{l_{0}} \pi_{1} L_{1}^{l_{1}} \pi_{2} \\
& \text { Type }\left(L_{0}\right)=(<,<) \\
& \text { Type }\left(L_{1}\right)=(<,<) \\
& \Delta_{\mathcal{B}}\left(L_{0}\right) \cdot x=\Delta_{\mathcal{B}}\left(L_{1}\right) \cdot y \\
& S\left(L_{0}\right) \geq S\left(L_{1}\right) \\
& \underline{l_{0}-x>0} \\
& \rho=\pi_{0} L_{0}^{l_{0}-x} \pi_{1} L_{1}^{l_{1}+y} \pi_{2}
\end{aligned}
$$

Each rule consists of conditions (lines above the bar) and a conclusion $\rho$, which is a path, below the bar. Their names indicate which type of loops are 


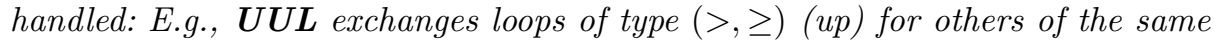
type on the left.

We say a rule is applicable to a sane path $\pi$ if there are $0<x, y, l_{0}, l_{1} \in \mathbb{N}$ and two different loops $L_{0}$ and $L_{1}$ such that all conditions are satisfied. In this case the rule can rewrite $\pi$ to $\rho$, its conclusion and we say $\rho$ is the result of applying the rule to $\pi$.

Example 5. Consider Example 2 again: The substitution suggested there is an application of the rule $\boldsymbol{U} \boldsymbol{U} \boldsymbol{L}$ to the path $\pi=\left(t_{0} t_{1} t_{2}\right)\left(t_{3} t_{4}\right)^{9} t_{5}\left(t_{6}\right)^{20}$, where $L_{0}=\left(t_{0} t_{1} t_{2}\right), L_{1}=\left(t_{3} t_{4}\right)$ and $x=y=8$. The result is a reduced witness for $\left(p 0, p^{\prime} 10\right)$ of length 50 . Shorter reduced witnesses for $\left(p 0, p^{\prime} 10\right)$ exist, for example $\left(t_{0} t_{1} t_{2}\right)^{6} t_{5} t_{6}^{16}$, but because of their different loop structure, these cannot be obtained from $\pi$ by applying rewriting rules, as these do not change the structure, i.e., which loops occur and in which order, of a path. This means that our rules do not necessarily preserve minimality of witnesses.

In the next two Lemmas 6 and 7, we show that the rewriting rules preserve witnesses and that continuous rule application must eventually terminate.

Lemma 6. If $\pi$ is a sane witness for $\left(p m, p^{\prime} m^{\prime}\right)$ and $\rho$ is the result of applying one of the rules to $\pi$, then $\rho$ is also a sane witness for $\left(p m, p^{\prime} m^{\prime}\right)$.

Proof. Each rule only modifies the number of times some loops are iterated, and never completely removes a loop. Therefore, sane paths are always rewritten to other sane paths.

Let's say we rewrite $\pi=\pi_{0} L_{0}^{l_{0}} \pi_{1} L_{1}^{l_{1}} \pi_{2}$ to $\rho$. The key observation is that the conditions of the rule imply that we can always decompose the paths $\pi$ and $\rho$ into $\pi=\alpha \gamma$ and $\rho=\beta \gamma$, s.t. $\Delta_{\mathcal{B}}(\alpha)=\Delta_{\mathcal{B}}(\beta)$ and $\Delta_{\mathcal{A}}(\alpha) \leq \Delta_{\mathcal{A}}(\beta)$. By monotonicity (Lemma 1) and the assumption that $\pi$ is a witness, it is therefore sufficient to show that the result $\rho$ is still enabled in the initial position $\left(p m, p^{\prime} m^{\prime}\right)$. We proceed by case distinction for the used rule.

$\boldsymbol{U} \boldsymbol{U L}$. Since $\pi$ is a witness, its prefix $\alpha=\pi_{0} L_{0}^{l_{0}} \pi_{1} L_{1}^{l_{1}}$ must be enabled in $\left(p m, p^{\prime} m^{\prime}\right)$ and because Type $\left(L_{0}\right)=(>, \geq)$, so is the prefix $\beta=\pi_{0} L_{0}^{l_{0}+x} \pi_{1} L_{1}^{l_{1}-y}$ of the result $\rho$. Assume that $\left(p m, p^{\prime} m^{\prime}\right) \stackrel{\alpha}{\longrightarrow}\left(q n, q^{\prime} n^{\prime}\right)$ and $\left(p m, p^{\prime} m^{\prime}\right) \stackrel{\beta}{\longrightarrow}\left(q \hat{n}, q^{\prime} n^{\prime}\right)$. The condition $S\left(L_{0}\right) \geq S\left(L_{1}\right)$ of the rule implies that $\hat{n} \geq n \geq \Gamma\left(\pi_{2}\right)$ and therefore that $\rho$ is enabled in $\left(p m, p^{\prime} m^{\prime}\right)$.

$\boldsymbol{U U \boldsymbol { R }}$. The prefix $\pi_{0} L_{0}^{l_{0}-x}$ of $\pi$ must be enabled and since the last condition of the rule demands that $l_{0}-x>\left|\pi_{1} L_{1}\right|$, so is the path $\pi_{0} L_{0}^{l_{0}-x} \pi_{1} L_{1}$. The fact that Type $\left(L_{1}\right)=(>, \geq)$, means that also $\pi_{0} L_{0}^{l_{0}-x} \pi_{1} L_{1}^{l_{1}+y}$ and therefore the result $\rho$ is enabled in $\left(p m, p^{\prime} m^{\prime}\right)$.

UD . Type $\left(L_{1}\right)=(<,<)$ implies $S\left(L_{1}\right)<\infty$. Since $S\left(L_{0}\right)<S\left(L_{1}\right)$, we know that $S\left(L_{0}\right)<\infty$ and hence $\Delta_{\mathcal{B}}\left(L_{0}\right)>0$. The path $\pi_{0} L_{0}^{l_{0}-x}$ is a prefix of $\pi$ and is therefore enabled in $\left(p m, p^{\prime} m^{\prime}\right)$. As $l_{0}-x \geq\left|\pi_{1}\right|$ by assumption, we get that

$$
m+\Delta_{\mathcal{A}}\left(\pi_{0} L_{0}^{l_{0}-x}\right) \geq l_{0}-x \geq\left|\pi_{1}\right| \geq \Gamma\left(\pi_{1}\right)
$$


and similarly, by $\Delta_{\mathcal{B}}\left(L_{0}\right)>0$,

$$
m^{\prime}+\Delta_{\mathcal{B}}\left(\pi_{0} L_{0}^{l_{0}-x}\right) \geq l_{0}-x \geq\left|\pi_{1}\right| \geq \Gamma^{\prime}\left(\pi_{1}\right) .
$$

This means that the prefix $\beta=\pi_{0} L_{0}^{l_{0}-x} \pi_{1}$ of $\rho$ is enabled in $\left(p m, p^{\prime} m^{\prime}\right)$. Let us now consider the prefix $\alpha=\pi_{0} L_{0}^{l_{0}-x} L_{0}^{x} \pi_{1} L_{1}^{y}$ of $\pi$. Because $\Delta_{\mathcal{B}}\left(L_{0}\right) \cdot x=$ $-\Delta_{\mathcal{B}}\left(L_{1}\right) \cdot y$ we get $\Delta_{\mathcal{B}}(\alpha)=\Delta_{\mathcal{B}}(\beta)$. By $S\left(L_{0}\right)<S\left(L_{1}\right)$ we obtain that $\Delta_{\mathcal{A}}(\alpha) \leq$ $\Delta_{\mathcal{A}}(\beta)$. Because $\pi=\alpha L_{1}^{l_{1}-y} \pi_{2}$ is a witness for $\left(p m, p^{\prime} m^{\prime}\right)$, we can apply Lemma 1 to conclude $\rho=\beta L_{1}^{l_{1}-y} \pi_{2}$ must be a witness for $\left(p m, p^{\prime} m^{\prime}\right)$.

$\boldsymbol{D D} \boldsymbol{L}$. We know that $m+\Delta_{\mathcal{A}}\left(\pi_{0} L_{0}^{l_{0}}\right)+\Delta_{\mathcal{A}}\left(\pi_{1}\right) \geq \Gamma\left(L_{1}^{l_{1}}\right)$, because $\pi$ is enabled in $\left(p m, p^{\prime} m^{\prime}\right)$. As $L_{1}$ is a type $(<,<)$ loop we also know that $\Delta_{\mathcal{A}}\left(L_{1}\right)<0$. Therefore, $\Gamma\left(L_{1}^{l_{1}}\right) \geq l_{1}$ and

$$
m+\Delta_{\mathcal{A}}\left(\pi_{0} L_{0}^{l_{0}}\right) \geq l_{1}-\Delta_{\mathcal{A}}\left(\pi_{1}\right) .
$$

Assume towards a contradiction that $m+\Delta_{\mathcal{A}}\left(\pi_{0} L_{0}^{l_{0}}\right)<\Gamma\left(L_{0}^{x} \pi_{1}\right)$. This means that

$$
m+\Delta_{\mathcal{A}}\left(\pi_{0} L_{0}^{l_{0}}\right)<\Gamma\left(L_{0}^{x}\right)+\left|\pi_{1}\right| \leq\left|L_{0}\right| \cdot x+\left|\pi_{1}\right| .
$$

This, together with Eq. (12) yields $l_{1}-\Delta_{\mathcal{A}}\left(\pi_{1}\right)<\left|L_{0}\right| \cdot x+\left|\pi_{1}\right|$ and thus $l_{1}<$ $\left|L_{0}\right| \cdot x+2\left|\pi_{1}\right|$ which contradicts the condition that $l_{1}>\left|L_{0}\right| \cdot x+2\left|\pi_{1}\right|$. Hence, $m+\Delta_{\mathcal{A}}\left(\pi_{0} L_{0}^{l_{0}}\right) \geq \Gamma\left(L_{0}^{x} \pi_{1}\right)$. By the same argument we get that $m^{\prime}+\Delta_{\mathcal{B}}\left(\pi_{0} L_{0}^{l_{0}}\right) \geq$ $\Gamma^{\prime}\left(L_{0}^{x} \pi_{1}\right)$. So the prefix $\beta=\pi_{0} L_{0}^{l_{0}+x} \pi_{1}$ of $\rho$ is enabled in $\left(p m, p^{\prime} m^{\prime}\right)$. Consider the prefix $\alpha=\pi_{0} L_{0}^{l_{0}} \pi_{1} L_{1}^{y}$ of $\pi$. By the assumption that $\Delta_{\mathcal{B}}\left(L_{0}^{x}\right)=\Delta_{\mathcal{B}}\left(L_{1}^{y}\right)$ we get that $\Delta_{\mathcal{B}}(\alpha)=\Delta_{\mathcal{B}}(\beta)$. Because of $S\left(L_{0}\right)<S\left(L_{1}\right)$ we get $\Delta_{\mathcal{A}}\left(L_{0}^{x}\right) \geq \Delta_{\mathcal{A}}\left(L_{1}^{y}\right)$ and therefore that $\Delta_{\mathcal{A}}(\alpha) \leq \Delta_{\mathcal{A}}(\beta)$. By Lemma 1 we conclude that the path $\rho=\beta L_{1}^{l_{1}-y} \pi_{2}$ is a witness for $\left(p m, p^{\prime} m^{\prime}\right)$.

$\boldsymbol{D D} \boldsymbol{R}$. Let $\alpha=\pi_{0} L_{0}^{l_{0}} \pi_{1}$ and let $\left(p m, p^{\prime} m^{\prime}\right) \stackrel{\alpha}{\longrightarrow}\left(q n, q^{\prime} n^{\prime}\right)$. Due to the type of $L_{0}$ and because $\pi$ is a witness, we know that the prefix $\beta=\pi_{0} L_{0}^{l_{0}-x} \pi_{1} L_{1}^{y}$ of $\rho$ is enabled in $\left(p m, p^{\prime} m^{\prime}\right)$. Since $\Delta_{\mathcal{B}}\left(L_{0}\right) \cdot x=\Delta_{\mathcal{B}}\left(L_{1}\right) \cdot y$, we get that $\left(p m, p^{\prime} m^{\prime}\right) \stackrel{\beta}{\longrightarrow}\left(q \hat{n}, q^{\prime} n^{\prime}\right)$ for some $\hat{n} \in \mathbb{N}$. The condition $S\left(L_{0}\right) \geq S\left(L_{1}\right)$ of the rule implies that $\Delta_{\mathcal{A}}\left(L_{0}^{x}\right) \leq \Delta_{\mathcal{A}}\left(L_{1}^{y}\right)<0$, and therefore that $\hat{n} \geq n$. We conclude that the path $L_{1}^{l_{1}} \pi_{2}$ is enabled in $\left(q r, q^{\prime} r^{\prime}\right)$ and therefore that $\rho=\pi_{0} L_{0}^{l_{0}-x} \pi_{1} L_{1}^{l_{1}+y} \pi_{2}$ is enabled in $\left(p m, p^{\prime} m^{\prime}\right)$ as required.

Lemma 7. Any sequence of successive applications of rules to a given path $\pi$ must eventually terminate.

Proof. Consider a $\pi$ to wich we apply the rewriting rules. W.l.o.g. assume $\pi$ is sane, as otherwise no rule is applicable by definition. The decomposition of $\pi$ is the sequence

$$
\operatorname{Dec}(\pi)=\left(\pi_{0}, L_{0}, l_{0}\right)\left(\pi_{1}, L_{1}, l_{1}\right) \ldots\left(\pi_{k}, L_{k}, l_{k}\right) \pi_{k+1}
$$

in $\left(E^{*} \times E^{*} \times \mathbb{N}\right)^{*} E^{*}$ such that $\pi=\pi_{0} L_{0}^{l_{0}} \pi_{1} L_{1}^{l_{1}} \ldots \pi_{k} L_{k}^{l_{k}} \pi_{k+1}$, where $k \leq F_{0}$ and for all indices $0 \leq i \leq k$, 
1. $L_{i}$ is a loop,

2. $\pi_{i}$ is acyclic,

3. for any two transitions $t \in \pi_{i}$ and $t^{\prime} \in L_{i}$ with $\operatorname{target}(t)=\operatorname{target}\left(t^{\prime}\right)$ holds that $\operatorname{target}\left(L_{i}\right)=\operatorname{target}(t)$.

The last condition demands that any loop $L_{i}$ shares exactly one node with the acyclic path $\pi_{i}$ it succeeds and thus ensures that the decomposition of a path is unique.

As no application of a rule completely removes all occurrences of loops nor introduces new ones nor touches the intermediate paths, we observe that rule applications only change the exponents $l_{i}$ in the decomposition of the path.

Based on the order of loops in the decomposition of $\pi$, and their potential for rule application, we now define a notion of weights for paths, and show that these weights have to strictly decrease along a well-order whenever a rule is applied.

Let $\left(L_{0}, L_{1}, \ldots, L_{k}\right)$ be the sequence of loops that occur in the decomposition of $\pi$. Let us fix some linear order $\prec$ on $\left\{L_{0}, L_{1}, \ldots, L_{k}\right\}$ that satisfies the following conditions for any two different loops $L_{i}, L_{j}$ with $i<j$.

1. If Type $\left(L_{i}\right)=$ Type $\left(L_{j}\right)=(>, \geq)$ and $S\left(L_{i}\right) \geq S\left(L_{j}\right)$ then $L_{i} \prec L_{j}$.

2. If Type $\left(L_{i}\right)=$ Type $\left(L_{j}\right)=(>, \geq)$ and $S\left(L_{i}\right)<S\left(L_{j}\right)$ then $L_{i} \succ L_{j}$.

3. If Type $\left(L_{i}\right)=$ Type $\left(L_{j}\right)=(<,<)$ and $S\left(L_{i}\right)<S\left(L_{j}\right)$ then $L_{i} \prec L_{j}$.

4. If Type $\left(L_{i}\right)=$ Type $\left(L_{j}\right)=(<,<)$ and $S\left(L_{i}\right) \geq S\left(L_{j}\right)$ then $L_{i} \succ L_{j}$.

Surely, such a linearization exists, as the conditions above only restrict $\prec$ between loops of the same type and slopes are linearly ordered. Consider the permutation $\sigma: \mathbb{N}_{\leq k} \rightarrow \mathbb{N}_{\leq k}$ given by $\sigma(i)<\sigma(j) \Longleftrightarrow L_{i} \prec L_{j}$. The weight of $\pi$ is

$$
W(\pi)=\left(l_{\sigma(k)}, l_{\sigma(k-1)}, \ldots, l_{\sigma(0)}\right) \in \mathbb{N}^{k+1} .
$$

The weight of $\pi$ is the ordered tuple of exponents $l_{i}$ of loops that occur in $\pi$. Because rules do not change the order of loop occurrences, the path before and after applying a rule have comparable weights. The very definition of weights ensures that rule applications must strictly reduce the weight of a path.

Claim. If $\rho$ is the result of applying a rewriting rule to $\pi$ then $W(\rho) \sqsubset l e x W(\pi)$ where $\sqsubset_{l e x}$ is the lexicographic extension of the pointwise ordering of tuples of naturals.

Assume the decompositions of $\pi, \rho$ are

$$
\begin{aligned}
& \operatorname{Dec}(\pi)=\left(\pi_{0}, L_{0}, l_{0}\right)\left(\pi_{1}, L_{1}, l_{1}\right) \ldots\left(\pi_{k}, L_{k}, l_{k}\right) \pi_{k+1} \text { and } \\
& \operatorname{Dec}(\rho)=\left(\pi_{0}, L_{0}, l_{0}^{\prime}\right)\left(\pi_{1}, L_{1}, l_{1}^{\prime}\right) \ldots\left(\pi_{k}, L_{k}, l_{k}^{\prime}\right) \pi_{k+1} .
\end{aligned}
$$

We show for every type of rule that if the occurrences of loop $L_{i}$ increase then those of some loop $L_{j}$ with $L_{i} \prec L_{j}$ strictly decrease.

If the rule used to derive $\rho$ was $\boldsymbol{U} \boldsymbol{U} \boldsymbol{L}$ then $l_{i}^{\prime}=l_{i}+x$ and $l_{j}^{\prime}=l_{j}-y$ for some $i<j, 0<x, y$ and type $(>, \geq)$ loops $L_{i}, L_{j}$ with $S\left(L_{i}\right) \geq S\left(L_{j}\right)$. By condition $1)$ in the definition of $\prec$ we get $L_{i} \prec L_{j}$. 
For rule $\boldsymbol{U} \boldsymbol{U} \boldsymbol{R}$ we know $l_{i}^{\prime}=l_{i}-x$ and $l_{j}^{\prime}=l_{j}+y$ for some $0<x, y$ and type $(>, \geq)$ loops $L_{i}, L_{j}$ with $S\left(L_{i}\right)<S\left(L_{j}\right)$. By condition 2) in the definition of $\prec$, we get $L_{i} \succ L_{j}$.

For rule $\boldsymbol{D} \boldsymbol{D} \boldsymbol{L}$ we know $l_{i}^{\prime}=l_{i}+x$ and $l_{j}^{\prime}=l_{j}-y$ for type $(<,<)$ loops $L_{i}, L_{j}$ with $S\left(L_{i}\right)<S\left(L_{j}\right)$. By condition 3) in the definition of $\prec$, we know $L_{i} \prec L_{j}$.

For rule $\boldsymbol{D D R}$ we know $l_{i}^{\prime}=l_{i}-x$ and $l_{j}^{\prime}=l_{j}+y$ for some $0<x, y$ and type $(<,<)$ loops $L_{i}, L_{j}$ with $S\left(L_{i}\right)>S\left(L_{j}\right)$. So condition 4) in the definition of $\prec$, implies $L_{i} \succ L_{j}$.

Lastly, if the rule used to derive $\rho$ was $\boldsymbol{U D}$ we immediately see that $l_{i}^{\prime}<l_{i}$ and $l_{j}^{\prime}<l_{j}$, which implies the claim.

Lemmas 6 and 7 allow us to focus on witnesses that are reduced, i.e., which are sane and to which none of the rewriting rules is applicable. We can now derive bounds on the multiplicities of loops in reduced paths.

Lemma 8. Let $\pi=\pi_{0} L_{0}^{l_{0}} \pi_{1} L_{1}^{l_{1}} \pi_{2}$ be a reduced path where $L_{0}, L_{1}$ are loops occurring with multiplicities $l_{0}>0$ and $l_{1}>0$.

1. If Type $\left(L_{0}\right)=$ Type $\left(L_{1}\right)=(>, \geq)$ and $S\left(L_{0}\right) \geq S\left(L_{1}\right)$ then $l_{1} \leq|V|$

2. If Type $\left(L_{0}\right)=$ Type $\left(L_{1}\right)=(>, \geq)$ and $S\left(L_{0}\right)<S\left(L_{1}\right)$ then $l_{0} \leq\left|\pi_{1}\right|+2|V|$

3. If Type $\left(L_{0}\right)=$ Type $\left(L_{1}\right)=(<,<)$ and $S\left(L_{0}\right)<S\left(L_{1}\right)$ then $l_{1}<|V|^{2}+2\left|\pi_{1}\right|$

4. If Type $\left(L_{0}\right)=$ Type $\left(L_{1}\right)=(<,<)$ and $S\left(L_{0}\right) \geq S\left(L_{1}\right)$ then $l_{0}<|V|$

5. If Type $\left(L_{0}\right)=(>, \geq)$, Type $\left(L_{1}\right)=(<,<)$ and $S\left(L_{0}\right) \leq S\left(L_{1}\right)$ then $l_{0} \leq$ $\left|\pi_{1}\right|+|V|$ or $l_{1} \leq|V|$.

Proof. The fourth condition of any rule is satified e.g. by $x=\Delta_{\mathcal{B}}\left(L_{1}\right)$ and $y=\Delta_{\mathcal{B}}\left(L_{0}\right)$. So if $0<x, y \in \mathbb{N}$ is the smallest satisfying pair we know $x, y \leq|V|$. The bounds are now easily derived by contradiction:

1. If $l_{1} \geq|V|$ then $l_{1}-y \geq l_{1}-|V|>0$ and rule $\boldsymbol{U} \boldsymbol{U} \boldsymbol{L}$ is applicable.

2. If $l_{0}>\left|\pi_{1}\right|+2|V|$ then $l_{0}-x>\left|\pi_{1}\right|+2|V|-x \geq\left|\pi_{1}\right|+\left|L_{1}\right| \geq\left|\pi_{1} L_{1}\right|$ and therefore rule $\boldsymbol{U} \boldsymbol{U} \boldsymbol{R}$ is applicable.

3. If $l_{1} \geq|V|^{2}+2\left|\pi_{1}\right|$ then $l_{1} \geq\left|L_{0}\right| \cdot x+2\left|\pi_{1}\right|$ and $l_{1}-y \geq l_{1}-|V|>0$, so rule $\boldsymbol{D} \boldsymbol{D} \boldsymbol{L}$ is applicable.

4. If $l_{0}>|V|$ then $l_{0}-x>0$, so rule $\boldsymbol{D D R}$ is applicable.

5. If $l_{1}>|V|$ and $l_{0}>\left|\pi_{1}\right|+|V|$, then $l_{1}-y>0, l_{0}-x>0$ and $l_{0}-x>\left|\pi_{1}\right|$, so rule $\boldsymbol{U D}$ is applicable.

In each case we conclude that one of the rules is applicable, which contradicts the assumption that $\pi$ is reduced.

Finally, we are ready to prove Theorem 1.

Theorem 1. Fix a $D O C N \mathcal{A}$, a complete $D O C N \mathcal{B}$, and let $K \in \mathbb{N}$ be the number of nodes in their product. There is a bound $c \in \mathbb{N}$ that depends polynomially on $K$, such that the following holds for any two processes pm and qn of $\mathcal{A}$ and $\mathcal{B}$. If $T(p m) \nsubseteq T(q n)$, then there is a witness for (pm,qn) that is either no longer than $c$ or has one of the following forms: 
1. $\pi_{0} L_{0}^{l_{0}} \pi_{1}$, where $L_{0}$ is a loop of type $(\geq,<)$ and $\pi_{0}, \pi_{1}$ are no longer than $c$,

2. $\pi_{0} L_{0}^{l_{0}} \pi_{1} L_{1}^{l_{1}} \pi_{2}$, where $L_{0}$ and $L_{1}$ are loops of type $(>, \geq)$ and $(<,<)$ with $S\left(L_{0}\right)>S\left(L_{1}\right)$ and $\pi_{0}, \pi_{1}, \pi_{2}$ are no longer than $c$,

3. $\pi_{0} L_{0}^{l_{0}} \pi_{1}$, where $L_{0}$ is a loop of type $(<,<)$ and $\pi_{0}, \pi_{1}$ are no longer than $c$,

where in all cases, the number of iterations $l_{0}, l_{1} \in \mathbb{N}$ are polynomial in $K$ and the initial counter-values $m$ and $n$ of the given processes.

Proof. We show that we can sufficiently increase the bound $c$ such that whenever $T(p m) \nsubseteq T(q n)$ but no witness exists that is shorter than $c$ or of forms 1) or 2), then there must be a witness of form 3$)$.

Assume $T(p m) \nsubseteq T(q n)$ and consider a reduced witness $\pi$, that is minimal in length: no shorter witness is reduced. Recall that this also means that $\pi$ is sane: it is of form described in Eq. (9). By monotonicity (Lemma 3) and because $\pi$ is of minimal length among the reduced witnesses, we see that it cannot contain loops of type $(\leq, \geq)$. Moreover, because $\pi$ is not of form 1$)$, we can safely assume that $\pi$ it contains only loops of types $(>, \geq)$ and $(<,<)$. This is because if a witness contains two or more different type $(\geq,<)$ loops, then there exists another (sane) witness, that only unfolds the first such loop. Relaxing the bound on the length of paths between loops to $F_{1}:=F_{0}\left(2|V|+|V|^{2}\right)$, we can write $\pi$ as

$$
\pi=\pi_{0} L_{0}^{l_{0}} \pi_{1} L_{1}^{l_{1}} \ldots \pi_{k} L_{k}^{l_{k}} \pi_{k+1}
$$

where $k \leq F_{0}$, all $\left|\pi_{i}\right|<F_{1}$ and the number of iterations of loop $L_{i}$ is $l_{i}>|V|$.

Consider a block $\pi_{\text {pos }}=L_{i}^{l_{i}} \pi_{i+1} L_{i+1}^{l_{i+1}} \pi_{i+2} \ldots \pi_{j} L_{j}^{l_{j}}$ that is part of the decomposition above, such that all loops are type $(>, \geq)$. If for indices $i \leq x<y \leq j$ we have $S\left(L_{x}\right) \geq S\left(L_{y}\right)$, then by Lemma 8.1 we get $l_{y} \leq|V|$. Therefore, $\pi_{\text {pos }}$ can be rewritten to the form

$$
\pi_{\text {pos }}=L_{i}^{l_{i}} \pi_{i+1} L_{i+1}^{l_{i+1}} \pi_{i+2} \ldots \pi_{j} L_{j}^{l_{j}} \pi_{j+1}
$$

where the lengths of $\pi_{i}$ are bounded by $F_{2}:=F_{0} \cdot\left(|V|^{2}+F_{1}\right)$ and the slopes of loops are strictly increasing: $S\left(L_{x}\right)<S\left(L_{y}\right)$ for any two indices $i \leq x<y \leq j$. By Lemma 8.2 this means that $l_{x} \leq\left|\pi_{x+1}\right|+2|V| \leq F_{2}+2|V|=: F_{3}$. We conclude that the prefix $\pi^{\prime}=L_{i}^{l_{i}} \pi_{i+1} L_{i+1}^{l_{i+1}} \pi_{i+2} \ldots \pi_{j-1} L_{j-1}^{l_{j-1}}$ is no longer than $(j-i) \cdot\left(|V| \cdot F_{3}+F_{2}\right)$ and therefore

$$
\pi_{\text {pos }}=\pi^{\prime} L_{j}^{l_{j}} \pi_{j+1}
$$

where $\left|\pi^{\prime}\right|$ is bounded by $F_{4}:=F_{0}\left(|V| \cdot F_{3}+F_{2}\right)$ and $\left|\pi_{j+1}\right|$ by $F_{2}$.

We continue to show by a similar argument that we can bound the number of iterations of all but the most productive loop in a block consisting of only decreasing (type $(<,<)$ ) loops. Consider a block $\pi_{n e g}=L_{i}^{l_{i}} \pi_{i+1} L_{i+1}^{l_{i+1}} \pi_{i+2} \ldots \pi_{j} L_{j}^{l_{j}}$ that is part of the decomposition in Eq. (17), where all loops are type $(<,<)$. If $S\left(L_{x}\right) \geq S\left(L_{y}\right)$ for some indices $i \leq x<y \leq j$, then by Lemma 8.4 we know $l_{y}<|V|$. This means that $\pi_{\text {neg }}$ is of the form

$$
\pi_{n e g}=\pi_{i} L_{i}^{l_{i}} \pi_{i+1} L_{i+1}^{l_{i+1}} \pi_{i+2} \ldots \pi_{j} L_{j}^{l_{j}} \pi_{j+1}
$$


where all $\pi_{i}$ have lengths bounded by $F_{2}$ and $S\left(L_{x}\right)<S\left(L_{y}\right)$ for any two indices $i \leq x<y \leq j$. By Lemma 8.3 we get $l_{y} \leq|V|^{2}+2\left|\pi_{x}\right| \leq|V|^{2}+2 F_{2}=: F_{3}^{\prime}$ and conclude that the suffix $\pi^{\prime \prime}=\pi_{i+1} L_{i+1}^{l_{i+1}} \pi_{i+2} \ldots \pi_{j} L_{j}^{l_{j}} \pi_{j+1}$ is no longer than $(j-i) \cdot\left(|V| \cdot F_{3}^{\prime}+F_{2}\right)$. Therefore, $\pi_{\text {neg }}$ is of the form

$$
\pi_{n e g}=\pi_{i} L_{i}^{l_{i}} \pi^{\prime \prime}
$$

where $\pi_{i}$ is bounded by $F_{2}$ and $\pi^{\prime \prime}$ by $F_{4}^{\prime}:=F_{0}\left(|V| \cdot F_{3}^{\prime}+F_{2}\right)$.

Eqs. (19) and (21) characterize the form of maximal subpaths of the witness $\pi$ in Eq. (17), along which the type of loops does not change. They allow us to write $\pi$ as

$$
\pi=\pi_{0} L_{0}^{l_{0}} \pi_{1} L_{1}^{l_{1}} \pi_{2} \ldots \pi_{k} L_{k}^{l_{k}} \pi_{k+1}
$$

where for all indices $0 \leq i<k$ :

1. $\pi_{i}$ is no longer than $F_{5}:=F_{3}+F_{3}^{\prime}+F_{4}+F_{4}^{\prime}$.

2. All $l_{i}>|V|$.

3. Consecutive loops $L_{i}$ and $L_{i+1}$ have different types.

4. If loops $L_{i}, L_{j}$ for $0 \leq i<j \leq k$ have the same type then $S\left(L_{i}\right)<S\left(L_{j}\right)$.

In the remainder of this proof, we further increase the polynomial bound for the gaps $\pi_{i}$ between the loops; this allows to conclude that $\pi$ contains at least one type $(<,<)$ loop and finally, that $\pi$ is of form 3$)$.

Observe that if all loops $L_{i}$ in Eq. (22) are of type $(>, \geq)$ then the witness is already of form $\pi=\pi_{0} L^{l} \pi_{1}$ as in Eq. (19), where $\pi_{0}, \pi_{1}$ are short and $L$ is the most effective loop. In this case, consider the run

$$
(p m, q n) \stackrel{\pi_{0} L^{l}}{\longrightarrow}\left(p^{\prime} m^{\prime}, q^{\prime} n^{\prime}\right)
$$

induced by the prefix $\pi_{0} L^{l}$. Because $\mathcal{B}$ is complete we know $\Delta_{\mathcal{B}}(\pi)=-n$. together with $\Delta_{\mathcal{B}}\left(\pi_{1}\right) \leq\left|\pi_{1}\right| \leq F_{5}$ we get $n^{\prime} \leq F_{5}$. Because $\Gamma_{\mathcal{A}}\left(\pi_{1}\right) \leq\left|\pi_{1}\right|$, we know that $l \leq\left|\pi_{1}\right| \leq F_{5}$ as otherwise, fewer iterations $l$ would result in a shorter witness and we assumed $\pi$ to be minimal in length. Hence, we could bound $\pi$ by $F_{6}:=F_{5}+|V| \cdot F_{5}+F_{5}$. So if we let $c \geq F_{6}$, our witness $\pi$ must contain type $(<,<)$ loops as it is assumed not to be no shorter than $c$.

Finally, fix an index $0 \leq x \leq k$ such that in Eq. (22), $L_{x}$ is a loop of type $(<,<)$ with most efficient decrease (minimal slope). That is, $\pi$ is of form

$$
\pi=\pi_{0} L_{x}^{l_{x}} \pi_{1} .
$$

We now bound both $\pi_{0}$ and $\pi_{1}$ and thereby prove that $\pi$ is of form 3). We start with the suffix $\pi_{1}$.

If $L_{x}$ is the only loop of type $(<,<)$, we are done as then $\left|\pi_{1}\right| \leq F_{5}$. Suppose we have two indices $0 \leq y<y+2 \leq k$, where both $L_{y}$ and $L_{y+2}$ are type $(<,<)$. This means that $L_{y+1}$ is of type $(>, \geq)$ with $S\left(L_{y+1}\right)<S\left(L_{y+2}\right)$. By Lemma 8.5 and the fact that $l_{y+2}>|V|$ we know that $l_{y+1}<\left|\pi_{y+1}\right|+|V| \leq F_{6}$. So $\pi_{y+1} L_{y+1}^{l_{y+1}} \pi_{y+2}$ is no longer than $2 \cdot F_{5}+|V| \cdot F_{6}=: F_{7}$. Applying Lemma 8.3 
to $L_{y}$ and $L_{y+2}$ we get $l_{y+2} \leq|V|^{2}+2 \cdot F_{7}=: F_{8}$ and thus $\pi_{y+1} L_{y+1}^{l_{y+1}} \pi_{y+2} L_{y+2}^{l_{y+2}}$ is no longer than $F_{9}:=F_{5}+\left(|V| \cdot F_{6}\right)+F_{5}+\left(|V| \cdot F_{8}\right)$. Now the above argument can be repeated for any successive pair of type $(<,<)$ loops in $\pi_{1}$ of which there are at most $F_{0}$. So, $\left|\pi_{1}\right|<F_{0} \cdot F_{9}$.

To bound the prefix $\pi_{0}$ in Eq. (24), we recall (point 3 above) that consecutive loops in Eq. (22) have different types and therefore $x \leq 1$. In case $x=0$, we immediately get $\left|\pi_{0}\right| \leq F_{5}$. If $x=1$, then $L_{0}$ is a type $(>, \geq)$ loop with $S\left(L_{0}\right)<S\left(L_{x}\right)$ and so by Lemma 8.5 and point 2), we get $l_{0} \leq\left|\pi_{1}\right|+|V|<F_{6}$. This means $\left|\pi_{0}\right| \leq 2 F_{5}+|V| \cdot F_{6}=F_{7}$.

We conclude that $c:=F_{9} \cdot F_{0}$ is sufficient to ensure that any witness $\pi$, longer than $c$ which is not of form 1) or 2) must have form 3 ). This completes our argument for the existence of witnesses in the claimed forms.

To see why $l_{0}$ and $l_{1}$ can always be bounded polynomially in $|V|$ and $m^{\prime}$ can be seen by looking at the types of the loops involved. For paths of form 1 and 3 , $L_{0}$ decreases the counter on the right at least once in every iteration. Since the value $m^{\prime}+\Delta_{\mathcal{B}}\left(\pi_{0}\right)$ before the first iteration is at most $m^{\prime}+c$, we have $l_{0} \leq m^{\prime}+c$.

Paths of the second form can be decomposed into a prefix $\pi_{0} L_{0}^{l_{0}}$ and a suffix $\pi_{1} L_{1}^{l_{1}} \pi_{2}$, which is a path of form 3 . Let $y_{0} \in \mathbb{N}$ be minimal such that the effect of the path $\gamma_{0}=\pi_{0} L_{0}^{0} \pi_{1} L_{1}^{y_{0}} \pi_{2}$, in which $L_{0}$ is not iterated at all is sufficient to reduce the initial value $m^{\prime}$ below 0 . That is, we have $m^{\prime}+\Delta_{\mathcal{B}}\left(\pi_{0} L_{0}^{0} \pi_{1} L_{1}^{y_{0}} \pi_{2}\right) \leq 0$. Note that as for forms 1 and 3, we can bound $y_{0}$ by $m^{\prime}+2 c$ and therefore, $\left|\gamma_{0}\right|$ is no larger than $3 c+|V| \cdot\left(m^{\prime}+2 c\right)$. This path might not be a witness because it is not enabled on the left side. However, because of the condition on the slopes, there are $x, y \leq|V|$ such that the effect of the loops satisfy

$$
\Delta_{\mathcal{B}}\left(L_{0}\right) \cdot x=-\Delta_{\mathcal{B}}\left(L_{1}\right) \cdot y \quad \text { and } \quad \Delta_{\mathcal{A}}\left(L_{0}\right) \cdot x>-\Delta_{\mathcal{A}}\left(L_{1}\right) \cdot y .
$$

This means, increasing the iterations of the loops $L_{0}$ and $L_{1}$ by $x$ and $y$, respectively, does not change the effect of the path on the right, but strictly increases the effect on the left. We increase the iterations $\left(l_{0}, l_{1}\right)=\left(0, y_{0}\right)$ in $\gamma_{0}$ as suggested above for $\Gamma\left(\gamma_{0}\right)<\left|\gamma_{0}\right|<3 c+|V| \cdot\left(m^{\prime}+2 c\right)$ times. The resulting path $\gamma_{1}=\pi_{0} L_{0}^{x_{1}} \pi_{1} L_{1}^{y_{1}} \pi_{2}$ is then surely witness, and iterates the loops not more than $x_{1}=3 c+|V| \cdot\left(m^{\prime}+2 c\right)$ and $y_{1}=m^{\prime}+5 c+|V| \cdot\left(m^{\prime}+2 c\right)$ times. 